ARTICLE

DOI: $10.1038 / s 41467-018-03759-y$

\title{
Precisely printable and biocompatible silk fibroin bioink for digital light processing 3D printing
}

Soon Hee Kim¹, Yeung Kyu Yeon ${ }^{1}$, Jung Min Lee ${ }^{1}$, Janet Ren Chao ${ }^{2}$, Young Jin Lee ${ }^{1}$, Ye Been Seo ${ }^{1}$, Md. Tipu Sultan ${ }^{1}$, Ok Joo Lee ${ }^{1}$, Ji Seung Lee ${ }^{1}$, Sung-il Yoon ${ }^{3}$, In-Sun Hong ${ }^{4}$, Gilson Khang ${ }^{5}$, Sang Jin Lee (b) ${ }^{6}$, James J. Yoo ${ }^{6}$ \& Chan Hum Park ${ }^{1,7}$

Although three-dimensional (3D) bioprinting technology has gained much attention in the field of tissue engineering, there are still several significant engineering challenges to overcome, including lack of bioink with biocompatibility and printability. Here, we show a bioink created from silk fibroin (SF) for digital light processing (DLP) 3D bioprinting in tissue engineering applications. The SF-based bioink (Sil-MA) was produced by a methacrylation process using glycidyl methacrylate (GMA) during the fabrication of SF solution. The mechanical and rheological properties of Sil-MA hydrogel proved to be outstanding in experimental testing and can be modulated by varying the Sil-MA contents. This Sil-MA bioink allowed us to build highly complex organ structures, including the heart, vessel, brain, trachea and ear with excellent structural stability and reliable biocompatibility. Sil-MA bioink is well-suited for use in DLP printing process and could be applied to tissue and organ engineering depending on the specific biological requirements.

\footnotetext{
${ }^{1}$ Nano-Bio Regenerative Medical Institute, College of Medicine, Hallym University, Chuncheon 24252, Republic of Korea. ${ }^{2}$ School of Medicine, George Washington University, Washington, D.C. 20037, USA. ${ }^{3}$ Division of Biomedical Convergence, College of Biomedical Science, Kangwon National University, Chuncheon 24341, Republic of Korea. ${ }^{4}$ Department of Molecular Medicine, School of Medicine, Gachon University, Incheon 406-840, Republic of Korea. ${ }^{5}$ Department of BIN Convergence Technology, Department of Polymer Nano Science \& Technology and Polymer Materials Fusion Research Center, Chonbuk National University, Jeonju 54896, Republic of Korea. ${ }^{6}$ Wake Forest Institute for Regenerative Medicine, Wake Forest School of Medicine, Medical Center Boulevard, Winston-Salem, NC 27157, USA. ${ }^{7}$ Departments of Otorhinolaryngology-Head and Neck Surgery, Chuncheon Sacred Heart Hospital, School of Medicine, Hallym University, Chuncheon 24252, Republic of Korea. These authors contributed equally: Soon Hee Kim, Yeung Kyu Yeon, Jung Min Lee. Correspondence and requests for materials should be addressed to C.H.P. (email: hlpch@paran.com)
} 
R ecently, bioprinting technology has moved toward the goal to create more complex structures with different tissue components and intrinsic microvasculature ${ }^{1,2}$. Bioprinting permits cells, biomaterials, and bioactive molecules to be placed in a precise manner, so as to create a complex three-dimensional (3D) tissue structure for biological and clinical applications $s^{3,4}$. Bioprinting technologies can be classified ${ }^{3-5}$ into inkjet printing, extrusion printing (fused deposition modeling; FDM), lightassisted bioprinting ${ }^{6-11}$, including digital light processing (DLP) and laser-based printing (Table 1). Inkjet bioprinting that is similar to conventional two-dimensional inkjet printing has the advantages of being relatively low cost and capable of moderate printing speed $\left(\mathrm{mm} \mathrm{s}^{-1}\right)$; however, disadvantages include the inability to use high-viscosity materials and high cell density due to nozzle clogging and inability to construct $3 \mathrm{D}$ tissue structures $^{12}$. The extrusion bioprinter was developed by modifying the inkjet printer and uses an air pump or a screw plunger to dispense bioinks. Because of this design, the extrusion type printer is compatible with hydrogels of various viscosities, but larger mechanical stresses on the encapsulated cells from more viscous hydrogels and a relatively long printing time can reduce cell viability by $40-80 \%{ }^{3,13}$. In contrast, DLP bioprinter can overcome these limitations. They create models in a layer-by-layer fashion unlike other printing modalities through photopolymerization by ultraviolet (UV) light. As a result, DLP achieves high resolution (about $1 \mu \mathrm{m})$ and with rapid printing speed $\left(\sim 30 \mathrm{~min}, \mathrm{~mm}^{3} \mathrm{~s}^{-1}\right)$ regardless of the layer's complexity and area. In addition, DLP printing increases cell viability beyond $85-95 \%$ due to the short printing time and nozzle-free printing technique $e^{5,14,15}$.

The printable materials or bioinks need to satisfy several essential criteria in terms of printability, biocompatibility, and biomimetic properties, including structural and mechanical stability. All of these requirements are essential for long-term shape constancy ${ }^{7,16}$. Particularly, when using the DLP modality, the bioink must be able to deposit in a layer-by-layer fashion, create Z-layer definition, and be photocurable ${ }^{5}$.

Hydrogels, which form 3D crosslinked hydrated fibers, are suitable as a bioink in 3D bioprinting. They can be used as a cell matrix, and provide a mechanically supportive microenvironment, that can be modified to mimic native tissue and its extracellular matrix. There are only a few reported biomaterials capable of producing a hydrogel for 3D bioprinting, including fibrinogen, agarose, gelatin, hyaluronic acid, and alginate ${ }^{13,17}$. A mixture of chitosan and polyethylene glycol diacrylate (PEGDA) ${ }^{18}$, a mixture of pluronic diacrylate and hyaluronic acid methacrylate ${ }^{19}$, and a mixture of PEGDA and gelatin methacrylate (GelMA) hydrogel ${ }^{7}$ have been reported as potential bioinks for DLP printing. However, the hydrogels based on synthetic materials have inherently low cell adhesion abilities, and the natural material-based hydrogels have insufficient stiffness, thereby making it difficult to control the matrix's rigidity ${ }^{20}$. In DLP printing, the dynamics of the polymerization can be adjusted by changing the power of the light source, the printing rate, and the type and concentrations of the photoinitiators, however, ultimately, the biomaterial itself controls the printability and mechanical properties of the printed materials.

Silk fibroin (SF), a natural fibrous protein produced by Bombyx mori, has been used for a variety of biomedical and biotechnological applications, including as a wound dressing ${ }^{21,22}$, enzyme immobilization matrix ${ }^{23}$, vascular prosthesis, and structural implant ${ }^{24,25}$. SF can be processed into different forms and structures, including as a film, gel, membrane, powder, and porous sponges ${ }^{25-27}$ under all-aqueous processing conditions and has been applied to tissue engineering. For other purposes, SF has been incorporated with various materials ${ }^{28}$ through chemical modifications such as coupling reactions, amino-acid modifications, and grafting reactions depending on the particular application. Addition of methacrylate groups to the amine-containing side groups of a material can be used to make it light polymerizable into a hydrogel. A material's degradation time can also be tailored by varying the degree and location of methacrylation $^{29-31}$. We hypothesized that SF would be an excellent bioink for DLP 3D bioprinting through methacrylation. Until now, SF itself has not been used for DLP printing due to the absence of a crosslinkage site essential for photopolymerization and we do not believe that SF has ever been modified with methacrylate groups directly for light polymerization.

In this study, we demonstrate a technique to develop an effective bioink for DLP printing with chemically modified SF by glycidyl methacrylate (GMA) (Sil-MA). We evaluate the degree of methacrylation on SF modified by various GMA amounts and characterize its physical and mechanical properties depending on Sil-MA concentration relative to the potential for suturing. Furthermore, we demonstrate the biocompatibility of the Sil-MA hydrogels prepared by DLP printing and its printability for different organs with complex structures.

\section{Results}

Sil-MA characterization. The Sil-MA was synthesized by methacrylate substitution of the primary amines of SF (Fig. 1a). To find suitable GMA amount, Sil-MA was fabricated by adding GMA with various molar ratios from 141 to $705 \mathrm{mM}$ into SF solution (Fig. 1b). The resulting Sil-MA and Sil-MA hydrogel were characterized depending on the different GMA amounts. Modification of GMA on SF was confirmed through identification

\begin{tabular}{|c|c|c|c|c|c|c|c|c|}
\hline Printer & Bioink & Photoinitiator & Light source & $\begin{array}{l}\text { Mechanical } \\
\text { properties }\end{array}$ & $\begin{array}{l}\text { Target tissue } \\
\text { or structure }\end{array}$ & Cell type & Culture & Ref. \\
\hline STL & $\begin{array}{l}\text { PEGDA and } \\
\text { GelMA }\end{array}$ & $\begin{array}{l}\text { Eosin Y-based } \\
\text { photo initiator }\end{array}$ & Customized visible light & $\begin{array}{l}\text { Young's } \\
\text { modulus (60 } \\
\mathrm{kPa} \text { ) }\end{array}$ & Hydrogel & $\mathrm{NIH} 3 \mathrm{~T} 3$ cells & In vitro (5 days) & 7 \\
\hline STL & $\begin{array}{l}\text { Methacrylated } \\
\text { PEG-co-PDP }\end{array}$ & LAP & $\begin{array}{l}\text { Visible light projection } \\
\text { SLA (3200 lumens) }\end{array}$ & $\begin{array}{l}38 \mathrm{kPa} \\
\text { (stiffness) }\end{array}$ & $\begin{array}{l}\text { Cell-laden } \\
\text { hydrogel }\end{array}$ & HUVEC cells & In vitro (10 days) & 10 \\
\hline
\end{tabular}

LAP lithium phenyl-2,4,6 trimethylbenzoyl phosphinate, GelMA gelatin methacrylate, GMHA glycidyl metacrylate hyaluronic acid, PEGDA polyethylene glycol diacrylate, HA hyaluronic acid, PEG-co-PDP poly (ethylene glycol-co-depsipeptide), hiPSC-HPC human iPSC-derived hepatic progenitor cells, DLP digital light processing based bioprinter, STL stereolithography, LAB laser-assisted bioprinter 

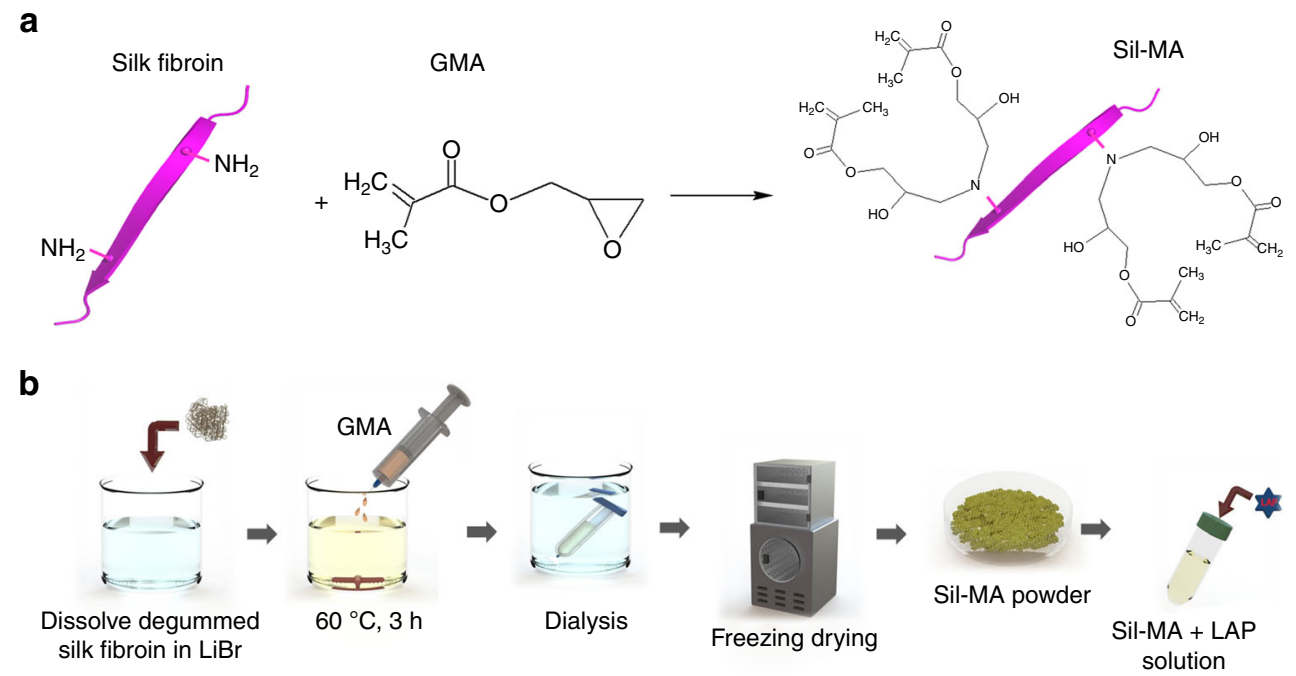

Fig. 1 Fabrication of chemically modified silk fibroin (SF) by glycidyl methacrylate (GMA) (Sil-MA) as pre-hydrogel. a Modification of SF molecule with GMA. SF is covalently immobilized with GMA, which is a donor of vinyl double bond as a UV-crosslinking site. $\mathbf{b}$ Schematic representation for methacrylation of SF. Degummed silk was dissolved in $9.5 \mathrm{M} \mathrm{LiBr} \mathrm{LiBr}$ and GMA was dropped into the solution with stirring for $3 \mathrm{~h}$ at $60^{\circ} \mathrm{C}$. It was dialyzed to remove salts in distilled water at room temperature for 4 days and then freeze-dried. Lithium phenyl(2,4,6-trimethylbenzoyl) phosphinate (LAP) was added to GMA-modified SF solution

of GMA-related peaks and SF-related peaks in Fourier-transform infrared spectroscopy (FT-IR) (Fig. 2a). The FT-IR of pure regenerated SF (RSF) was determined to correlate the changes, which occurred in the vibration modes of the Sil-MA. The spectra in both RSF and all Sil-MA samples showed peaks of amide I, II, and III at 1639,1512 , and $1234 \mathrm{~cm}^{-1}$, respectively. A weak band in Sil-MA groups was visible at $1238 \mathrm{~cm}^{-1}$, which indicates $\mathrm{CHOH}$ stretching of the alcohol group, which was produced after the opening of the epoxy group of the GMA. The other small changes detected in the Sil-MA specimens were at 951 and 1165 $\mathrm{cm}^{-}$, representing $\mathrm{CH}_{2}$ wagging stretching of methacrylate vinyl group in the GMA. These peaks gradually increased with greater quantities of GMA. The GMA functional groups were only slightly detectable or hidden by SF peaks because the molecular weight of GMA is much smaller than that of the SF.

The degree of methacrylation was evaluated and the resulting, abovementioned ring-opening of epoxy at GMA and nucleophilic addition reactions by primary amine on lysine depending on GMA amounts was proved by proton nuclear magnetic resonance ( ${ }^{1} \mathrm{H}$-NMR) spectroscopy (Fig. 2b). Specifically, we found that the characteristic resonance of the methacrylate vinyl group $(\delta=$ $6.2-6$ and 5.8-5.6 ppm) and the methyl group of GMA at $\delta=1.8$ $\mathrm{ppm}$ has appeared by addition of the GMA, and the integration of these peaks increased with the addition of greater GMA amounts. In addition, there was a gradual decrease of the lysine methylene signal at $\delta=2.9 \mathrm{ppm}$ with increasing quantities of GMA, which indicated modification of the lysine residues in SF $(22-42 \%$; Table 2). Lastly, measurable signals were found at $\delta=3.2-3.6$ $\mathrm{ppm}$ resulting from the hydrogen neighboring the $\mathrm{C}-\mathrm{N}$ bond.

The microstructures of the Sil-MA hydrogels were observed by scanning electron microscope (SEM; Fig. 2c). The SEM images revealed uniform and interconnected porous microstructures throughout all the samples. The pore size showed decreasing pattern with higher degrees of methacrylation and Sil-MA concentration (Supplementary Fig. 1). It has been previously reported that the degree of methacrylation affects the physical and mechanical properties of hydrogels, with higher methacrylation resulting in stiffer and tougher hydrogels and smaller pore sizes $^{31}$. Based on these results, a schematic representation illustrating the network formation was created (Fig. 2d).
Mechanical properties and water uptake of Sil-MA. Sil-MA hydrogel for mechanical testing was fabricated by the DLPmanufacturing machine using a Sil-MA slurry, including lithium phenyl(2,4,6-trimethylbenzoyl) phosphinate (LAP; Fig. 3a). A UV light intensity of $3.5 \mathrm{~mW} \mathrm{~cm}^{-2}$ for $3 \mathrm{~s}$ was sufficient to solidify the solution in each layer. We confirmed the mechanical suitability of Sil-MA hydrogel by obtaining stress-strain curves for compression and elongation (Supplementary Fig. 2a-b). The compressive elastic modulus increased with increasing Sil-MA concentration and strain (Fig. 3b). Every 10\% increment of SilMA concentration brought about 2.6-fold increases in the compressive stress (Fig. 3c). The compressive strain and the compressive stress at break increased according to the Sil-MA concentration. A particularly impressive outcome $(910 \mathrm{kPa}$ at break) was observed for the 30\%Sil-MA. The 30\%Sil-MA hydrogels were strong enough to support the weight of a kettle bell $(7 \mathrm{~kg})$ and returned to their original shape immediately after the kettle bell was removed (Fig. 3d). The higher concentration of Sil-MA hydrogels resulted in increased tensile strength and breaking elongation. Sil-MA's tensile elastic modulus was also increased with greater Sil-MA concentrations (Fig. 3e). The tensile strength of the $30 \%$ Sil-MA was 1.5 times higher, and the breaking elongation measured 1.6 times more than that of the $20 \%$ Sil-MA (Fig. 3f). We were able to suture using 30\%Sil-MA. The 3D-printed Sil-MA membrane was folded slightly and then sutured using simple interrupted suture without tearing (Fig. $3 \mathrm{~g}$ and Supplementary Movie 1). Furthermore, we were able to complete successfully an end-to-end anastomosis using 3Dprinted 30\%Sil-MA tracheal rings with subsequent continuity (Fig. 3h and Supplementary Movie 2).

In the water uptake test, specimen weight was increased with lengthening of the hydration time up to $5 \mathrm{~h}$, however, after that a constant weight was obtained for all Sil-MA hydrogels. It was calculated that the Sil-MA hydrogel had high water uptake 46 times greater with $10 \%$ Sil-MA hydrogel, 20 times greater with $20 \%$ Sil-MA hydrogel, and 11 times greater with 30\%Sil-MA hydrogel compared to each Sil-MA hydrogels' dry weight (Fig. 3i and Table 3). The volume expansion measurements in water (Fig. $3 \mathrm{j}$ and Table 3) also showed an increase in size of $150 \pm 0.9 \%$ for $20 \%$ Sil-MA hydrogel and $145.4 \pm 2.4 \%$ for $30 \%$ Sil-MA 
a

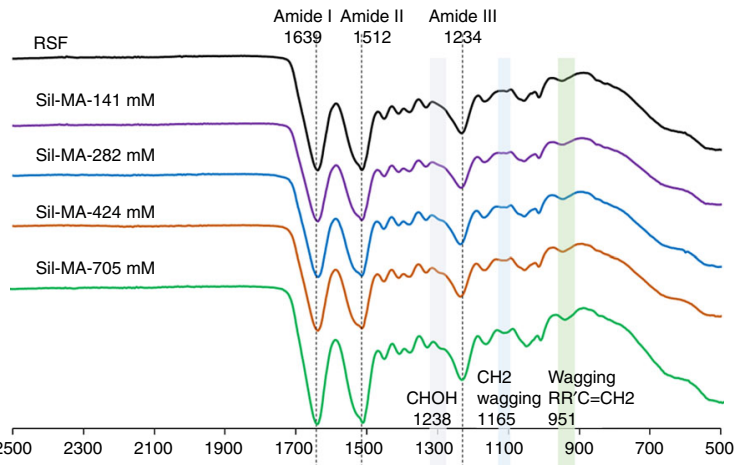

b

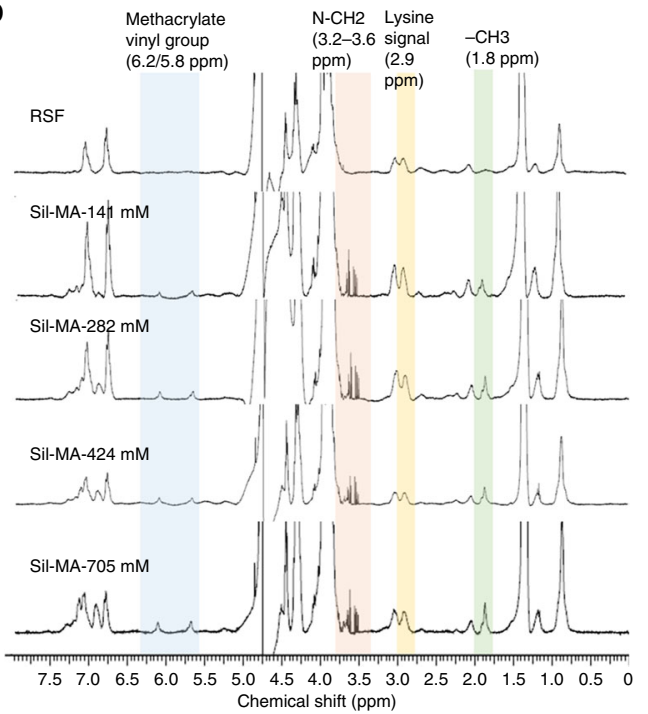

C
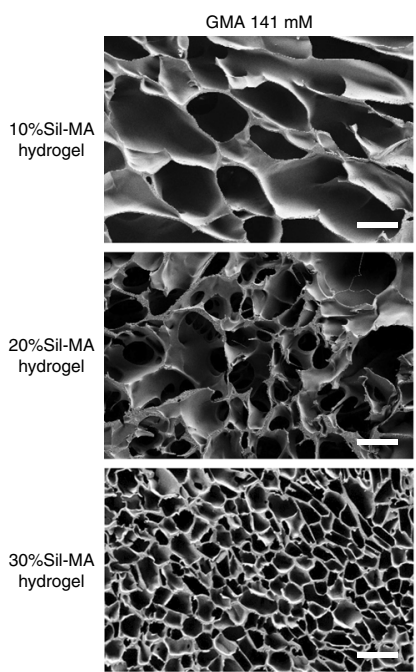
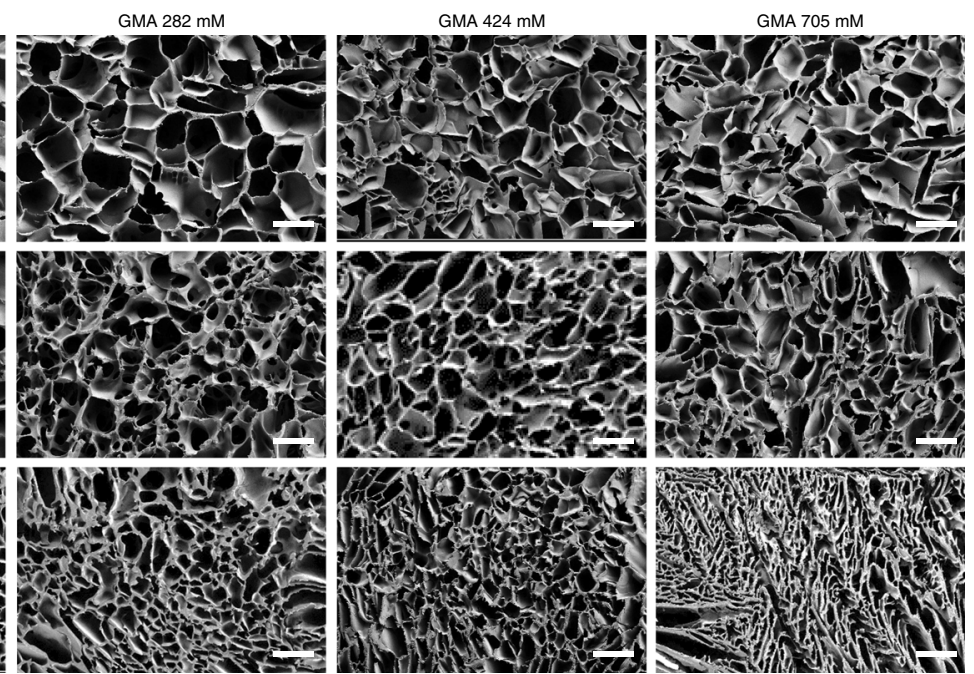

d

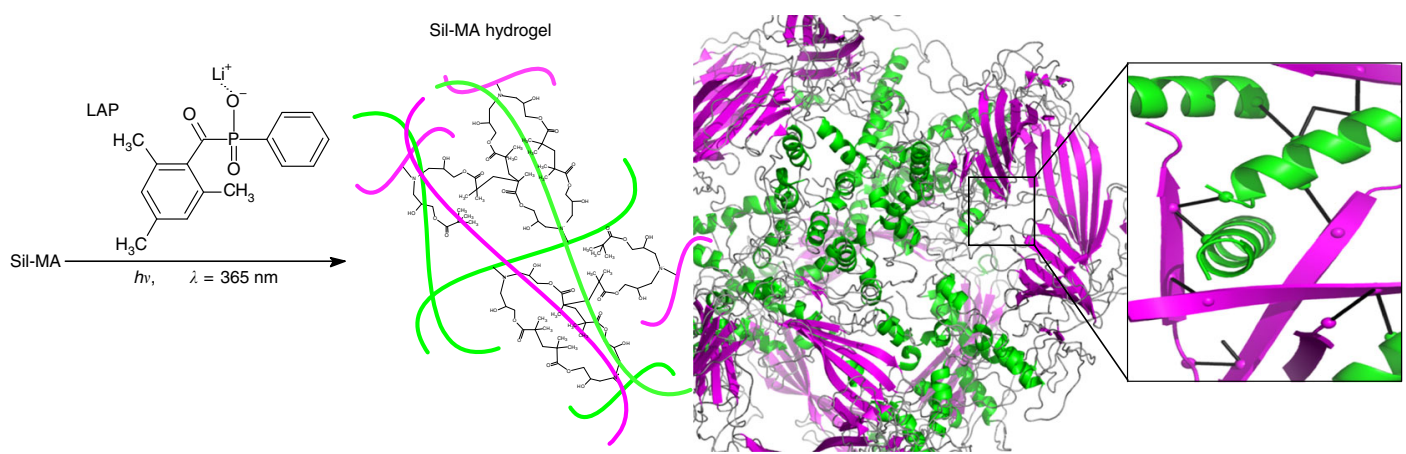

Fig. 2 Characterization of Sil-MA pre-hydrogel and Sil-MA hydrogel depending on methacrylation degrees. a FT-IR spectra and $\mathbf{b}{ }^{1} \mathrm{H}-\mathrm{NMR}$ spectra of unsubstituted SF and Sil-MA. In the FT-IR spectra, amide I $\left(1639 \mathrm{~cm}^{-1}\right)$, amide II $\left(1512 \mathrm{~cm}^{-1}\right)$, and amide III $\left(1234 \mathrm{~cm}^{-1}\right)$ shown in $\beta$-sheet of SF and spectra related with $\mathrm{GMA}$ such as $\mathrm{CHOH}, \mathrm{RR}{ }^{\prime} \mathrm{C}=\mathrm{CH}_{2}$ were found. The modification of lysine residues in $\mathrm{SF}$ with the increase of $\mathrm{GMA}$ was confirmed by the gradual decrease in the lysine signal at $\delta=2.9 \mathrm{ppm}$ and the increase in the methacrylate vinyl group signal at $\delta=6.2-6$ and 5.8-5.6 ppm, and the methyl group signal at $\delta=1.8 \mathrm{ppm}$. c FE-SEM images of Sil-MA hydrogels, presenting the effect of the degree of methacrylation and Sil-MA contents on the pore sizes of Sil-MA hydrogels. Scale bar represents $100 \mu \mathrm{m}$. d Reaction process for the photopolymerization of Sil-MA in the presence of LAP photoinitiator and 3D structure of Sil-MA hydrogel formed via digital light process (DLP) printing. With the presence of photoinitiator, the vinyl double bonds on GMA could react with each other intra-chain or between chains. SF chains themselves could entangle each other during photopolymerization. Green and magenta colors indicate alpha helix and beta sheet motif in the secondary structure of SF, respectively 


\begin{tabular}{|c|c|c|c|c|}
\hline Groups & Methacrylate vinyl group & Methyl group & Lysine & Methacrylation degree (\%) \\
\hline Sil-MA-141 mM & 0.23 & 0.55 & 1.11 & 22.4 \\
\hline Sil-MA-282 mM & 0.35 & 0.59 & 0.97 & 32.2 \\
\hline Sil-MA-424 mM & 0.75 & 0.87 & 0.83 & 42.0 \\
\hline
\end{tabular}

GMA was added at a concentration of $141,282,424$, and $705 \mathrm{mM}$ to $\mathrm{SF}$

hydrogel, both of which were lower than that of 10\%Sil-MA hydrogel $(175.2 \pm 6.1 \%)$. On the other hand, in medium without supplemental ingredient and in phosphate-buffered saline (PBS; $\mathrm{pH} 7.4$ ), their volume was not expanded as much as in water (Fig 3k, l).

Rheological properties of Sil-MA. To characterize the rheological properties of the Sil-MA hydrogels, an oscillatory rheological measurement was used. This test was carried out by varying the amplitude or frequency in a series of trials. For the amplitude sweep (Fig. $4 \mathrm{a}, \mathrm{b}$ ), the condition was set at $0.01-1 \%$ strain and a constant angular frequency $(10 \mathrm{~Hz})$. Storage modulus $\left(G^{\prime}\right)$ and loss modulus $\left(G^{\prime \prime}\right)$ represent the elastic part and the viscous part for Sil-MA hydrogels, respectively. Up to $1 \%$ shear strain, most hydrogels showed nearly constant values for $G^{\prime}$ and $G^{\prime \prime}$, which suggests that the structures in all specimens were undisturbed. The peak gradually rose with increasing Sil-MA concentration and the most marked peak was shown in the 30\%Sil-MA hydrogel. In the all groups, $G^{\prime}$ was larger than $G^{\prime \prime}$ by a factor of approximately 6-8 in the linear viscoelastic ranges regardless of the Sil-MA concentration, which meant that the Sil-MA gels behaved as elastomeric materials. Of note, the Sil-MA hydrogel closely resembled a solid given the phase angle $(\delta)$ of $6.4-9.1^{\circ}$ (Supplementary Table 1). The $G^{\prime}$ and $G^{\prime \prime}$ at $0.158-25.1 \mathrm{~Hz}$ of the frequency range and $1 \%$ deformations were measured. The properties of the material demonstrated minimal changes in stress at low frequencies, and greater changes at high frequencies. The increasing pattern of $G^{\prime \prime}$, which indicates weakly crosslinked Sil-MA in the network, was shown to a minimal degree during oscillation (Fig. 4c). The highest $G^{\prime}$ among Sil-MA groups was about $3 \mathrm{kPa}$ in the 30\%Sil-MA hydrogel (Fig. 4d).

The rheological properties of Sil-MA were monitored during the UV-curing process to assess the kinetics of the photocrosslinking reaction at $0.1 \%$ strain and $1 \mathrm{~Hz}$ frequency. They were measured both for the combination of varying photo-initiating concentration with constant Sil-MA content and for varying SilMA concentration under constant photo-initiating concentration. Figure 4e shows that Sil-MA hydrogels tended to become stiffer with increasing curing times, which meant that longer exposure to UV light resulted in greater stabilization of the hydrophobic domains when SF was methacrylated by GMA. The $G^{\prime}$ at $250 \mathrm{~s}$ corresponding to $3.5 \mathrm{~mJ} \mathrm{~cm} \mathrm{~cm}^{-2}$ of a UV energy density reached $130,241,642$, and $771 \mathrm{~Pa}$ in the condition with LAP $0.05 \%, 0.1 \%$, $0.2 \%$, and $0.4 \%$, respectively, and increased up to 642,10151 , and $25577 \mathrm{~Pa}$ as the Sil-MA concentration was increased from $10 \%$ to $20 \%$ and to $30 \%$, respectively (Fig. $4 \mathrm{f}$ ). Meanwhile, when UV was turned off after $4 \mathrm{~s}$ treatment in the $30 \%$ Sil-MA, the increase rate of $G^{\prime}$ was low. Supplementary Table 2 shows gel point depending on LAP or Sil-MA concentration. The starting point of gelation decreased from 101 to $41 \mathrm{~s}$ with increasing LAP concentration and rose from 74 to $137.5 \mathrm{~s}$ with increasing Sil-MA concentration.

The actual resolution and printability of Sil-MA via DLP printing. To get the actual resolution and accuracy of $3 \mathrm{D}$ DLP printing using Sil-MA, we measured the actual figure size after printing and determined the deviation by comparing the nominal diameters in both the horizontal and vertical planes. The observable features were formed when nominal $X$ and $Z$ dimensions were at least 100 and $300 \mu \mathrm{m}$, respectively (Supplementary Fig. 3a). Our system produced spatial accuracy with an average deviation of $66 \mu \mathrm{m}$ (circle diameter), $90 \mu \mathrm{m}$ (square width), and $142 \mu \mathrm{m}$ (height) (Supplementary Fig. 3b-d).

We chose the $30 \%$ Sil-MA for the printability test because it printed as designed for both pattern and size, as well as ease of handling after DLP printing (Supplementary Fig. 4). We mimicked various complex and difficult shapes to print as examples of the versatility of $30 \%$ Sil-MA using DLP printer. All printed structures mirrored the forms of the designed CAD images. Mesh style scaffolds with small $(\sim 700 \mu \mathrm{m})$ and highly interconnected pores visible to the naked eye were printed (Fig. 5a). In addition, a replica of the Eiffel Tower in miniature was accurately constructed by the DLP printer. Figure 5b, c shows that DLP printing using 30\%Sil-MA would be capable of constructing complex organ structures: ear auricle with helical fold; the cerebral sulcus and grooves; the lumen inside the trachea and joints on it; the heart with aorta and pulmonary artery/vein; and the lung with thoracic cavity and the vascular network structures with small caliber. In addition, when the printed products, including the brain and ear were distorted by pressure from the thumb and index finger, they returned to their original shape without residual deformity (Supplementary Movies 3 and 4). Finally, patency of the $1.6 \mathrm{~mm}$-sized blood vessels in the different models was demonstrated through perfusion.

Cytocompatability of Sil-MA hydrogel. Live/dead assay was carried out to evaluate the cytocompatibility of the Sil-MA hydrogel using NIH/3T3 fibroblast for potential cell encapsulation applications (Fig. 6a). Cells were suspended in Sil-MA solution $(10 \sim 30 \%)$ or commercial GelMA solution (as a control group, $10 \%)$. This cell suspension was used for printing by a DLP printer and the printed ones were cultivated for 14 days. Cells encapsulated inside Sil-MA hydrogels remained mostly viable regardless its concentration, and a mild aggregation was detected with increasing concentration of Sil-MA due to strong interaction between cells by decreasing pore sizes (Fig. 2c). The average pore sizes are inversely related to the concentration of hydrogel materials ${ }^{30}$. CCK-8 assay demonstrated that the cells grew well in the Sil-MA hydrogel and that the cell proliferation was as high in the $10 \%$ GelMA as in $30 \%$ Sil-MA (Fig. 6b).

Figure $6 \mathrm{c}-\mathrm{f}$ demonstrates cell distribution into the Sil-MA hydrogel through DLP printing. It was found that cells were distributed evenly over the printed constructs regardless their size and complexity of structure from four specimens. In the logo printing, the embossed logo (thickness $400 \mu \mathrm{m}$ ) against a bottom sheet was printed out clearly with even cell distribution (Supplementary Movie 5). Second, in brain construction, the cells labeled with green fluorescence were printed without 
a

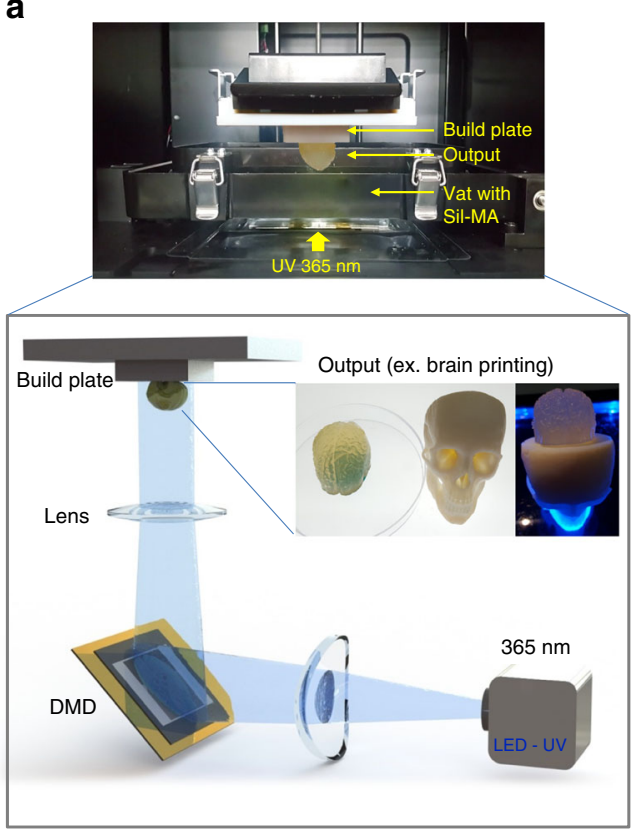

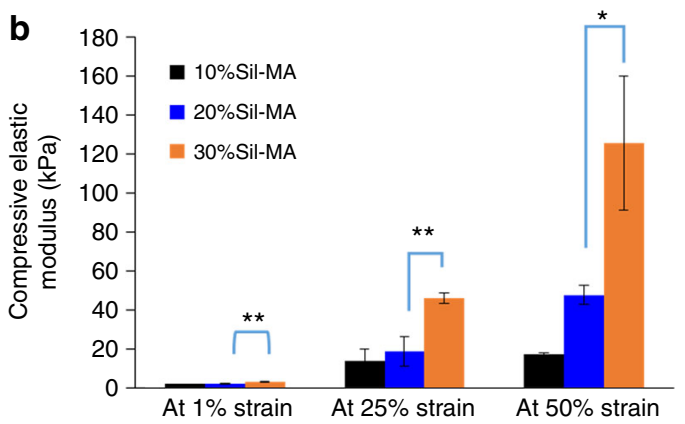
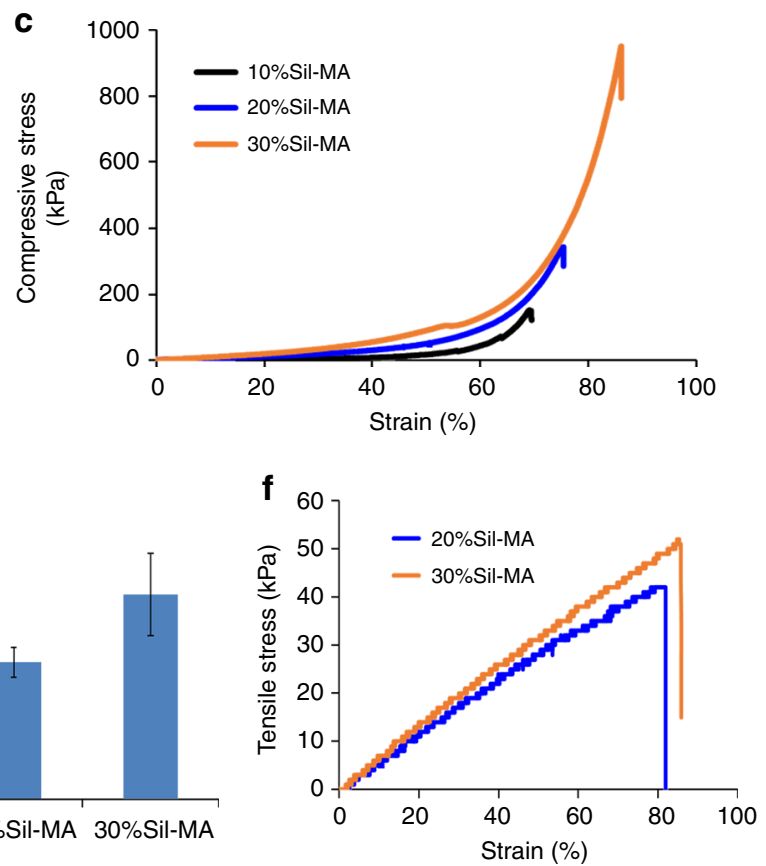
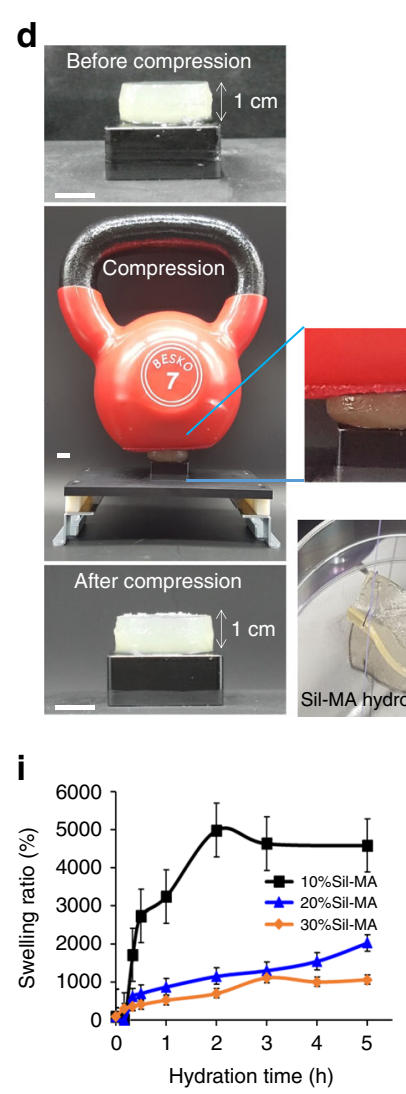

e

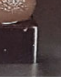

\section{g}

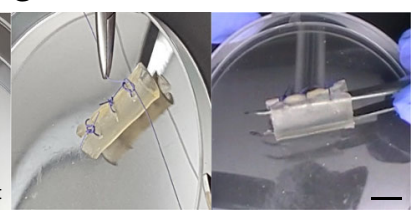

h

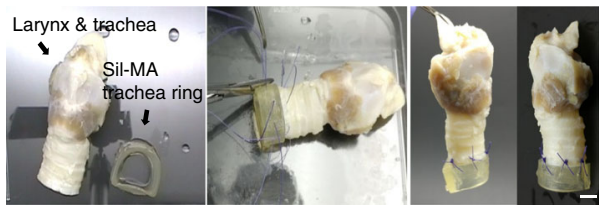

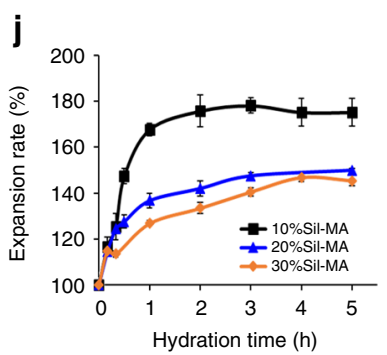
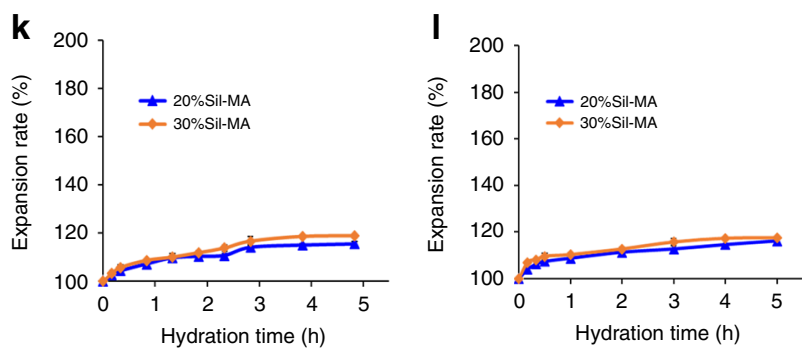

Fig. 3 Physical properties of Sil-MA hydrogel. a Schematic diagram of DLP bioprinting procedure using Sil-MA. Sil-MA-added LAP was printed in a layerby-layer style with DLP printer. Herein, the desired pattern was designed through CAD software and was sliced to a layer file before transferring it to the DLP system. DMD digital micromirror device. b-d Compression test for Sil-MA hydrogel with varied Sil-MA contents. b Compressive elastic modulus of Sil-MA hydrogel at different percentage strains. Significant differences are presented. ${ }^{\star} p<0.05$ and ${ }^{* *} p<0.005$ (two-sample $t$-test). c Representative compressive stress-strain curve and $\mathbf{d}$ the Sil-MA hydrogel was compressed by a kettle bell $(7 \mathrm{~kg})$ for 3 min and recovered hydrogel's initial shape after it was removed. e-h Tensile stress for Sil-MA hydrogel. e Tensile elastic modulus of Sil-MA hydrogel at $50 \%$ strain and $\mathbf{f}$ representative tensile stress-strain curve of Sil-MA hydrogel. $\mathbf{g}$ Suturing of folded lumen structure of 3D-printed Sil-MA membrane and $\mathbf{h}$ trachea end-to-end anastomosis of dog's larynx and trachea using Sil-MA 3D DLP-printed hydrogel. Scale bar indicates $1 \mathrm{~cm}$. i-I Swelling properties. i Water uptake of Sil-MA powder. Volume expansion rate of DLP product in $\mathbf{j}$ water, $\mathbf{k}$ medium, and I PBS ( $\mathrm{pH} 7.4)$. Data are presented as mean \pm s.d. The black line (/bar), blue line (/bar), and orange line (/bar) indicate $10 \%, 20 \%$, and $30 \%$ of Sil-MA hydrogel, respectively. Each assay was conducted in triplicate 
Table 3 Mechanical properties of Sil-MA hydrogel depending on Sil-MA contents

\begin{tabular}{|c|c|c|c|}
\hline Compressive strain at break (\%) & $69.5 \pm 0.5$ & $77.7 \pm 3.3$ & $80 \pm 5.1$ \\
\hline Tensile stress at break $(\mathrm{kPa})$ & ND & $52 \pm 4.3$ & $75 \pm 7.5$ \\
\hline Elongation at break (\%) & ND & $77.6 \pm 3.8$ & $124.2 \pm 41$ \\
\hline Tensile elastic modulus at $50 \%$ strain $(\mathrm{kPa})$ & ND & $9.7 \pm 1.0$ & $14.5 \pm 2.9$ \\
\hline Water content at $5 \mathrm{~h}$ from dried Sil-MA (\%) & $4580 \pm 480$ & $2026 \pm 202$ & $1059 \pm 121$ \\
\hline
\end{tabular}

obliteration of the small grooves and sulcus on it (Supplementary Movie 6). Third, in the logo printing model using cells labeled with two colors, cell position could be controlled as designed (Supplementary Movie 7). Rather than simply printing a one- to two-layer structure, we printed a colorful trachea of four layers with different colors on each layer with good integrity and even cell distribution (Supplementary Movie 8). Most notable is that cell distribution and clear printing as designed were accomplished although all the printing size was close to a tenth of a coffee bean.

Cartilage tissue engineering using Sil-MA hydrogel. For longterm biocompatibility of Sil-MA, we fabricated a ring-like cartilaginous trachea by DLP printer and tested it in vitro. Figure 7 shows in vitro result at each time point of $1,2,3$, and 4 weeks to demonstrate in vitro cartilage tissue formation. Cells were distributed evenly over the Sil-MA hydrogel and exhibited rounded morphology with lacunae embedded in basophilic extracellular matrix when the cell-laden Sil-MA hydrogel was stained using Hematoxylin and Eosin (HE). The pericellular region and interterritorial matrix region were densely stained by the typical red of Safranin-O indicating proteoglycan-rich matrix and by blue distinctive of Masson's Trichrome (MT) indicating the collagen matrix. Closely packed chondrocytes with lacunae were notably observed and the matrix around them was progressively stained over time. In the fluorescence microscopy examinations, the visible red signal demonstrated the presence of chondrocytes for up to 4 weeks in vitro, which was consistent with the histological data. We evaluated degradation rate of 30\%Sil-MA hydrogel with its potential value for DLP printing. Supplementary Fig. 5 shows degradability of $30 \%$ Sil-MA hydrogel with cells in vitro. Cellloaded Sil-MA hydrogel degraded gradually and exhibited a 50\% degradation rate at 4 weeks after cultivation. These results indicate that the Sil-MA hydrogel provided an excellent environment for the growth of chondrocytes and cartilage formation in vitro.

\section{Discussion}

DLP additive manufacturing has the potential to contribute to improved fabrication of complex structures in tissue engineering. However, DLP printing for tissue engineering would not be possible in the absence of photocrosslinkable bioinks possessing good mechanical strength and biocompatibility.

SF produced by $B$. mori is one of the strongest fibrous proteins in nature with biocompatibility and biodegradable properties. With that said, most of the silk materials developed from RSF solution have ordinary mechanical properties compared to native silk fibers due to the degumming and dissolution processes. Therefore, the RSF has been enhanced mechanically through various processing strategies, including increase of temperature, methanol/salt treatment, physically blended or chemically crosslinked with other polymers, and so on. Several studies have been tried to fabricate a photocrosslinked hydrogel based on SF by combining another materials introduced methacrylated groups (e.g., GelMA and poly(vinyl alcohol) methacrylate) ${ }^{32,33}$. However, there have not yet been studies of photocuring sites being introduced directly into the SF itself. Though one study about photocrosslinkable SF, which uses riboflavin as a photoinitiator and visible light as a light source ${ }^{34}$ has been reported, the visible light, which is needed for longer treatment (minutes), and has a high penetration ratio is not suitable for the DLP printer to create the detailed patterns required. Therefore, a more suitable modification method for SF is necessary for the use of the DLP technique.

Previously, we modified the timing of adding GMA to the SF. From this we found that the early addition of GMA into the SF solution produced the best Sil-MA through an unknown mechanism. This is different from the usual methacrylated gelatin (GelMA)-manufacturing method. We also found that final quality of Sil-MA depends on the reagent used for methacrylation. We applied both MA and GMA, reagents commonly used in methacrylation of gelatin, to SF. However, the crystallization of the SF during either the reaction or dialysis was observed when MA was applied to the SF. We speculated that one possible reason for this phenomenon is that a reaction by-product, methacrylic acid, reduces the $\mathrm{pH}$ of the SF solution during the reaction. As a result, the free amino groups of SF could become ionized, which hinders the reaction with $\mathrm{MA}^{35}$. With regards to the $\mathrm{pH}$ reduction, the protonation of the side chain carboxyl group that plays a principal role in determining gelation rate could be expected. This protonation would neutralize the carboxyl groups, reduce hydrophilicity, and decrease charge repulsion, which accelerate the hydrophobic interactions of the fibroin molecules and resultingly produce increased physical crosslinks and faster gelation $^{36}$. In contrast, application of GMA resulted in a stable reaction. We believe that the reaction using GMA was stable without acidic by-product through the mechanism of epoxide ring-opening (as a major mechanism) ${ }^{37}$. In addition, GMA could be to increase the reactions with the amino groups. Theoretically, two GMA molecules can react with one free amino group (Fig. 1a), while only one MA molecule reacts with one free amino group of the lysine residue ${ }^{38}$. Therefore, the use of GMA maximizes the number of vinyl groups on SF which contains only small quantities of lysine $(0.2 \mathrm{~mol} \%)^{28,39}$. GMA can also react with the hydroxyl and carboxyl groups in SF molecules. These functional groups can facilitate further methacrylation of SF through the transesterification mechanism (as a minor mechanism $)^{36}$, increasing the amount of vinyl methacrylate, and thus maximizing the crosslinking density of Sil-MA hydrogels ${ }^{40}$.

We varied the amount of GMA added to the SF solution to find the suitable methacrylation degree with minimal ingredients. SF $(\sim 350 \mathrm{kDa})$ has $0.2 \mathrm{~mol} \%$ lysine, which is likely the main site for the introduction of the GMA into $\mathrm{SF}^{28,39}$ In this reaction, SF would be coupled to the pendant active epoxide groups of the GMA by nucleophilic reaction between $-\mathrm{NH}_{2}$ moieties in the lysine on $\alpha$-helix or $\beta$-sheet of SF and epoxide groups ${ }^{41,42}$ Testing of the Sil-MA through FT-IR and ${ }^{1} \mathrm{H}-\mathrm{NMR}$ revealed that 

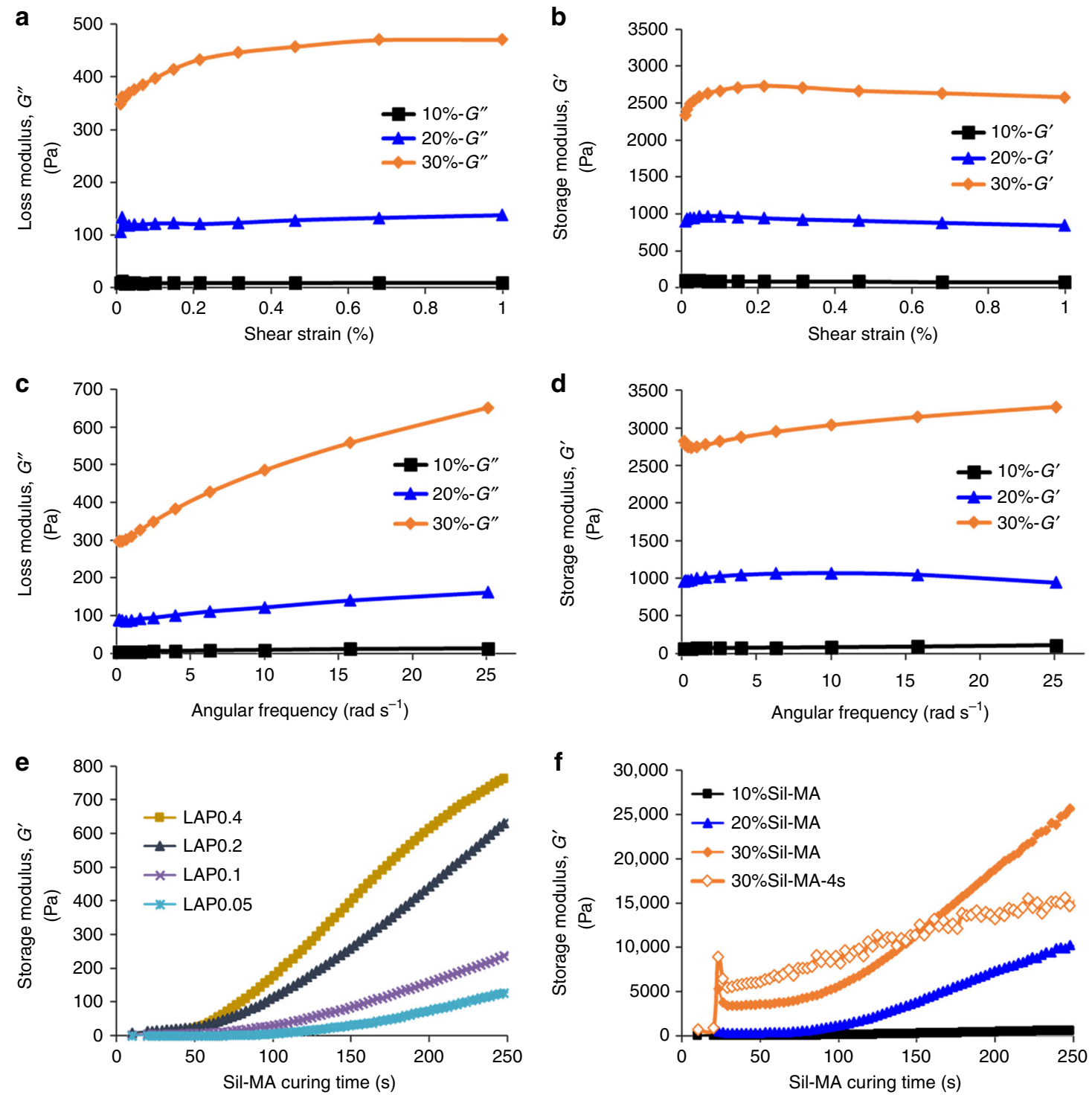

Fig. 4 Rheological analysis for Sil-MA hydrogel. a, b Strain dependency and $\mathbf{c}$, $\mathbf{d}$ frequency dependency of $\mathbf{a}, \mathbf{c}$ loss modulus $\left(G^{\prime \prime}\right)$ and $\mathbf{b}, \mathbf{d}$ storage modulus $G^{\prime}$ for Sil-MA hydrogels at different Sil-MA contents varied from 10 to $30 \%$. e, $\mathbf{f}$ In situ rheology during UV exposure. The effect of e LAP contents and $\mathbf{f}$ SilMA contents on the $G^{\prime}$ during UV exposure for 250 s except for the line with orange squares (30\%Sil-MA) exposed for only $4 \mathrm{~s}$. The black line, blue line, and orange line indicate $10 \%, 20 \%$, and $30 \%$ of Sil-MA hydrogel, respectively

GMA has been successfully grafted to the SF molecules (Fig. 2a, b). Overall, an increase in GMA concentration resulted in higher level of methacrylation of SF, which could be seen by the decrease in the lysine methylene group (Table 2). The degree of methacrylation affects the physical and mechanical properties of hydrogel such as stiffness, pore size, and durability of hydrogel ${ }^{31}$. Sil-MA hydrogel's mechanical properties can be tuned by adjusting GMA concentration depending on target tissue. However, there was no significant effect of increased amounts of GMA beyond $424 \mathrm{mM}$. We suggest that the ideal GMA concentration is from 282 to $424 \mathrm{mM}$ and it can be modified depending on applications, based on the required mechanical properties. In this study, $424 \mathrm{mM}$ of GMA showed maximum methacrylation rate and was selected for the various mechanical and cell viability studies. We assumed that unreacted lysine was shielded by the reacted sites as the reaction progressed and that the GMA would then react with other amino acids beside lysine. In fact, striking signals at $\delta=3.2-3.6 \mathrm{ppm}$ assigned to hydrogen neighboring $\mathrm{C}-\mathrm{N}$ bond were found. This result indicates that reactions occurred between the secondary amines in the amino acids of SF and GMA even though their reactivity is not as strong as in the primary amine. It has been reported that SF contains 1105 of the 5000 reactive amino acids, including lysine $0.2 \%$, arginine $0.3 \%$, asparagine $0.4 \%$, and glutamine $0.2 \%$ in its primary amino-acid group and histidine $0.1 \%$ and tryptophan $0.2 \%$ in the secondary group $^{28,39}$.

The fabricated Sil-MA allowed fabrication of the 3D structures using a DLP printer (Fig. 3a). A photoinitiator, LAP, was added to photopolymerize Sil-MA. LAP has greater water solubility and low cell cytotoxicity ${ }^{43}$. The photopolymerization reaction was initiated by LAP mixed with a Sil-MA solution and exposed to UV light of $365 \mathrm{~nm}$. LAP produces a free radical that attacks vinyl monomers of Sil-MA, which serve as a key linkage. It leads to radical-initiated chain polymerization since the surplus electrons will occur on the either end of the vinyl bond ${ }^{44,45}$.

Mechanical properties of the tissue-engineered product influences the cellular interactions and cell functions ${ }^{46}$. The capacity of 3D-printed scaffolds to resist compressive loads is important 

CAD

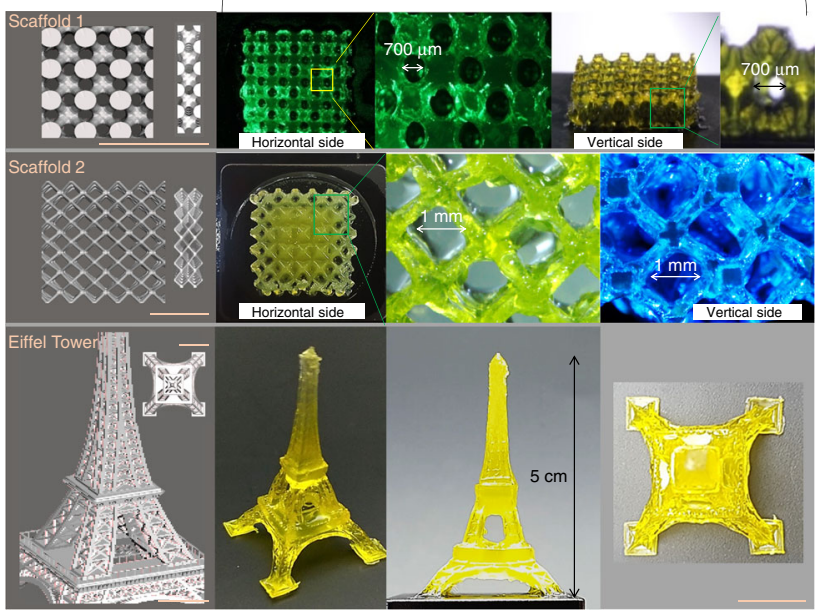

b

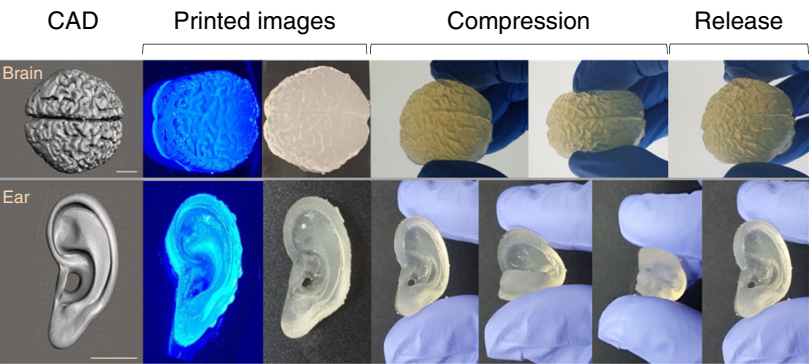

C CAD Printed images at various angles

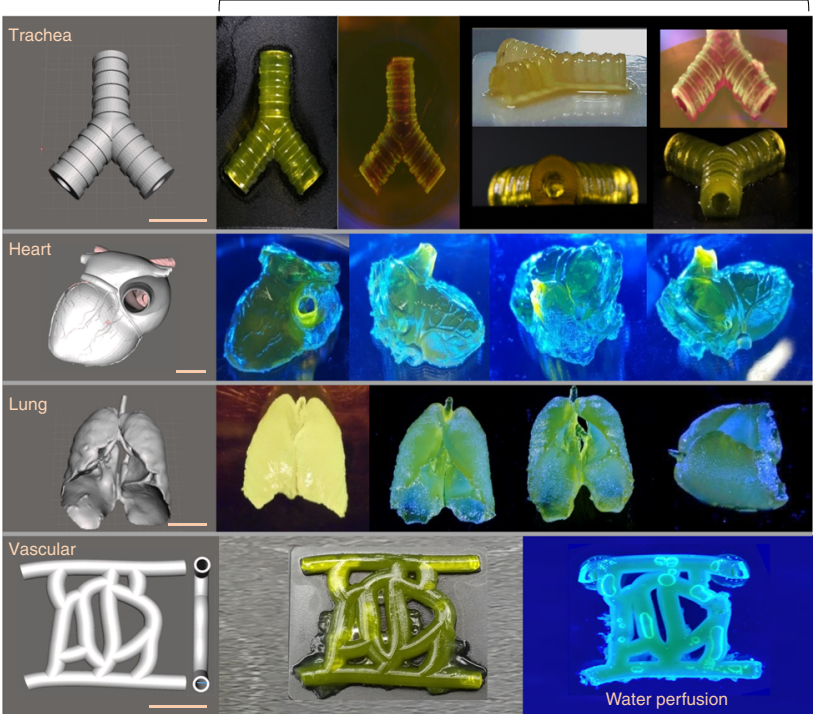

Fig. 5 Printability of 30\%Sil-MA using DLP printer. a Porous scaffold and Eiffel Tower imitation; (I) CAD images depicting scaffolds and Eiffel Tower and $(r)$ printed images. Printed scaffolds had small pores around $\sim 700 \mu \mathrm{m}$ and Eiffel Tower had small holes and grid on the surface. $\mathbf{b}$ Ear and brain mimicked shape; (I) CAD images depicting the ear and brain and ( $r$ ) printed images. Printed products were not damaged when they were compressed by fingers tightly and they were back to their original shape when relaxed his fingers. c Trachea, heart, lung, and vessel mimicked shape; (I) CAD images depicting the trachea, heart, lung, and vessel and $(r)$ printed images at various angles. Printed products by DLP using Sil-MA showed complex structure reflecting their $C A D$ images, including veins, arteries, folds, and holes. Scale bar indicates $1 \mathrm{~cm}$ given the frequent forces on the various structures and organs of the body. In this study, increasing the Sil-MA concentration resulted in enhancement of mechanical characteristics. Here, 30\% Sil-MA showed the strongest mechanical strength and excellent resiliency following distortion among groups (Fig. 3b-d). The compressive strength of 30\%Sil-MA hydrogel was about 10 times higher compared to PCL blended gelatin hydrogel as a synthetic/ natural hybrid scaffold $(75-94 \mathrm{kPa})^{47}$. The compressive elastic modulus of $30 \%$ Sil-MA was about 1.5 times higher than that of $30 \%$ GelMA ( $88 \mathrm{kPa}$; Supplementary Fig. 6). Based on its elastic modulus, pure Sil-MA hydrogel could be applied to the creation of 3D-printed artificial mitral valve leaflets $(<100 \mathrm{kPa})^{48}$, smooth muscle $(6-10 \mathrm{kPa})$, and carotid artery $(84 \mathrm{kPa})$. Sil-MA hydrogel also produced structures with highly flexible and stretchable properties as the concentration of Sil-MA was increased (Fig. 3e-h). Therefore, Sil-MA could be applied to a variety of clinical applications based on its acceptable mechanical properties.

We expect that the improved mechanical properties could be associated with physical entanglement of SF molecules, in addition to the chemical methacrylation of the SF. First, as Sil-MA content increases, the higher concentration of Sil-MA macromolecules in the Sil-MA solution raises the chances of chemical linkage and entanglements among the macromolecules. Second, the stimuli from this physicochemical entanglement between SilMA molecules could promote secondary crystalline conformation ( $\beta$-sheet) more likely, generating added strength and new hydrophobic regions within the $\mathrm{SF}^{49,50}$. Based on these assumptions, possible linkages affecting the strength and resilience of the Sil-MA hydrogel were drawn as a 3D structure in Fig. $2 \mathrm{~d}$ and are described as follows: (1) covalent intra-chain linkages; (2) chain-chain linkages through introduced vinyl double bonds; and (3) physical entanglement between the multiple long chains of SF macromolecules in the hydrogel.

Water uptake ability of hydrogel is important from the point of view of the affinity of the manufactured materials on the adjacent tissues. At the same time, the retention of shape is also necessary for medical applications. Sil-MA hydrogel had excellent swelling properties, but they were decreased with increasing Sil-MA contents (Fig. 3i-l). This limitation of water uptake and volume expansion with increasing Sil-MA concentration could be associated with the increased mechanical strength mentioned above.

Rheological properties of DLP-printed Sil-MA hydrogel, such as loss and storage modulus and the correlation between Sil-MA concentration and photoinitiator catalyst in terms of the starting point for gelation were confirmed controlling for LAP and SilMA content (Fig. 4). Data showed that the Sil-MA hydrogel was close to solids through the phase angle (Supplementary Table 1). Commonly, the phase angle is related to the viscoelastic character of materials. The phase angle is zero for a purely elastic material (solid-like behavior) and $90^{\circ}$ for a purely viscous material (liquidlike behavior). This characteristic is important for the medical requirements that must be fulfilled when using Sil-MA as a tissue replacement substance ${ }^{51}$. It was noted that increases in LAP augmented Sil-MA content and advanced the gelation time; however, this approach should be limited to avoid possible cytotoxicity.

In the laboratory, organs and tissues with tubular (e.g., trachea and vascular networks) as well as solid organs (e.g., ear, brain, heart, and lung) were mimicked with good mechanical integrity using $30 \%$ Sil-MA in a 3D DLP printer (Fig. 5). Current bioprinting techniques have technical challenges in regards to facilitating vascularization, innervation, and viable cell distribution within complex 3D tissues. All of these characteristics are essential for biological and clinical application of $3 \mathrm{D}$ printing to tissue engineering. Most researchers today, in order to create the 
a

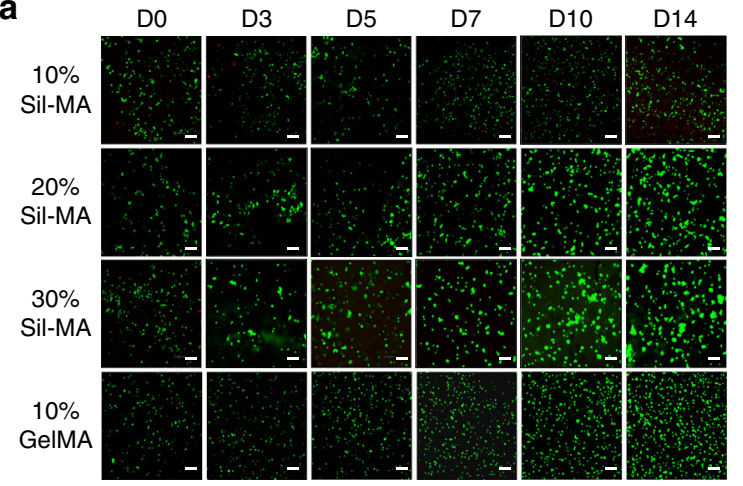

b

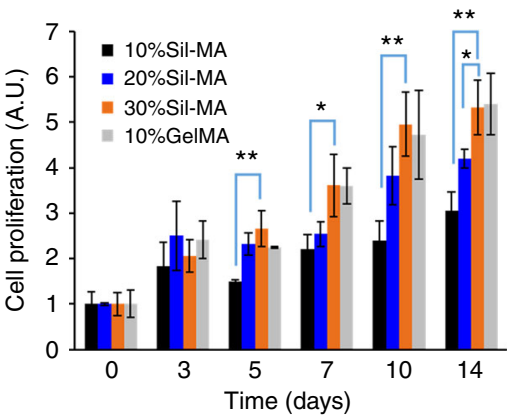

C

CAD

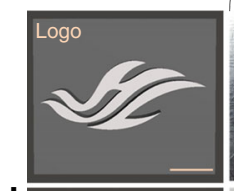

d

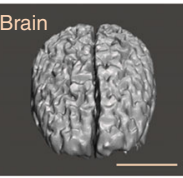

e

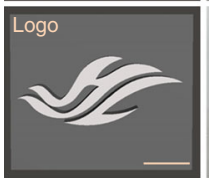

$\mathbf{f}$
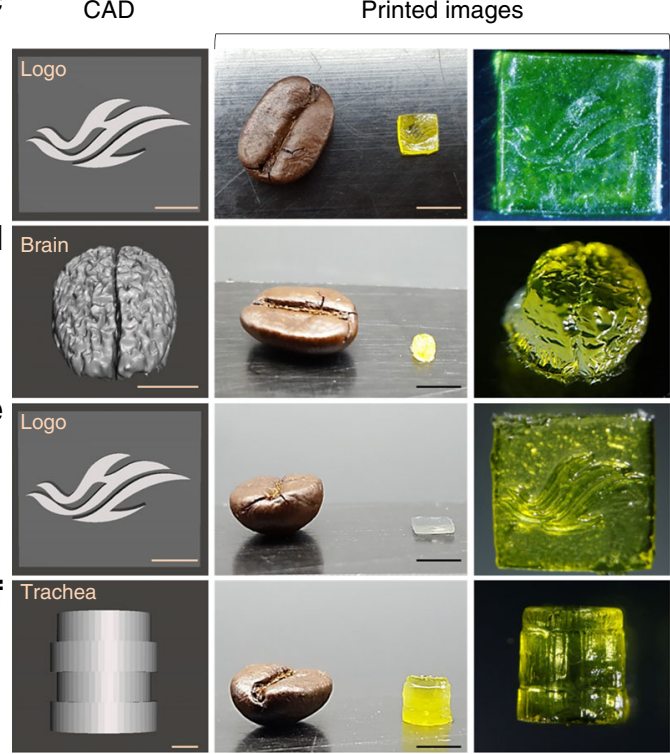

\section{FL images}

Merged images with CAD image

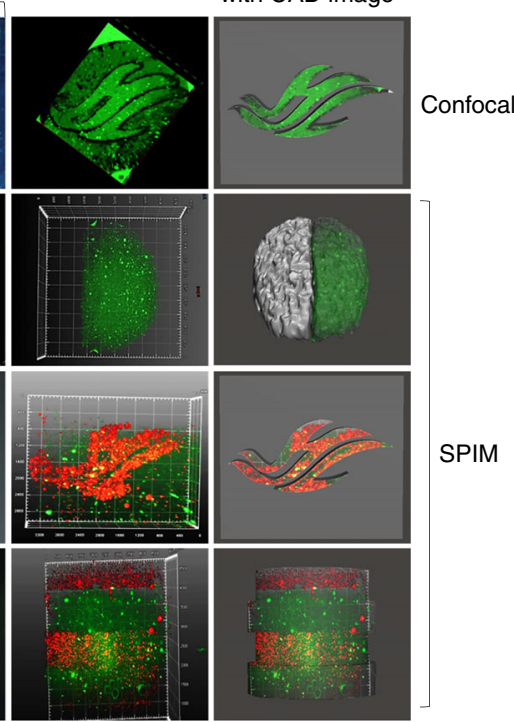

Fig. 6 Cytocompatibility of Sil-MA. a Live and dead assay; cell viability of encapsulated NIH/3T3 for 14 days (live cells in green and dead cells in red). Scale bar indicates $500 \mu \mathrm{m}$. b CCK-8 assay; cell proliferation in the hydrogel for 14 days. The $30 \%$ Sil-MA hydrogel showed the similar cell proliferation to that of commercial GelMA (10\%). * and ${ }^{\star \star}$ refer to statistically significant proliferation $p<0.05$ and $p<0.005$ (two-sample $t$-test), respectively, compared to $30 \%$ Sil-MA hydrogels. The black line, blue line, orange line, and gray line indicate 10\%, 20\%, and 30\% of Sil-MA hydrogel and $10 \%$ GelMA hydrogel,

respectively. c-f Cell distribution inside the Sil-MA hydrogel. Cells were distributed evenly in the hydrogels. The Sil-MA hydrogels with c a design of the letter $\mathrm{HL}$ (the logo of Hallym University) and $\mathbf{d}$ a shape of human brain were printed out with PKH67-labeled cells only. Also, the Sil-MA hydrogels with e the letter $\mathrm{HL}$ and $\mathbf{f}$ a shape of winding trachea were printed out with PKH67-labeled cells (green) and PKH26-labeled cells (red); (from left to right) CAD images, printed images, fluorescence images by c confocal or $\mathbf{d}-\mathbf{f}$ single plane illumination microscopy (SPIM) microscope, and merged images of

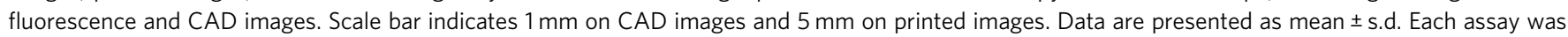
conducted in triplicate

lumen of the vasculature network, use extrusion printing with a sacrificial ink that creates the $3 \mathrm{D}$ spaces and then is resorbed after the outline is complete ${ }^{52}$. Alternatively, a coaxial nozzle can be used to make hollow fibers, but branched structures cannot be produced using this method ${ }^{53}$. In contrast, a DLP printing system with Sil-MA enables complex tubular structures with multiple branches (including capillary networks on the surface of the heart) to be created in one procedure without a complicated second set of steps. Our system facilitated structures that were not only stable, but layered up to $45-50 \mathrm{~mm}$ (e.g., brain and Eiffel Tower) significantly higher than what is possible with alternative hydrogels. This major advance in $3 \mathrm{D}$ printing results from the harmony between the unique properties of Sil-MA and the layering capability of DLP technology. The maximum height capable of being achieved with other hydrogels is limited to 3-10 $\mathrm{mm}$ using FDM and DLP modalities ${ }^{54-56}$.

DLP 3D-printed Sil-MA hydrogel could be a promising biomaterial in tissue engineering due to its excellent biocompatibility. Cells were not damaged because of rapid printing speed ( $3 \mathrm{~s}$ per layer) with accuracy, as a result, the cells grew well and were distributed equally over the hydrogel set, an outcome important for biological and clinical applications (Fig. 6). Furthermore, we demonstrated multilayer printing using different cells, replicating biological tissue, which consists of multi cell types. Appropriate viscosity is also important during 3D printing. DLP printing requires relatively low viscosity during the printing itself compared to extrusion techniques, but tissue engineering also needs higher viscosity to avoid deformation and collapse of the final product. Thirty percent Sil-MA with UV crosslinking fulfilled both of these requirements with excellent cell growth and detailed final structures. Also, the cell concentration of $1-2 \times 10^{7}$ cells per $\mathrm{mL}$ did not affect the integrity of the final construct. From the in vitro study using human chondrocyte, we were able to observe degradation of the 30\%Sil-MA hydrogel up to $50 \%$ for 4 weeks and the positive environment for chondrocytes in the hydrogel (Fig. 7). 


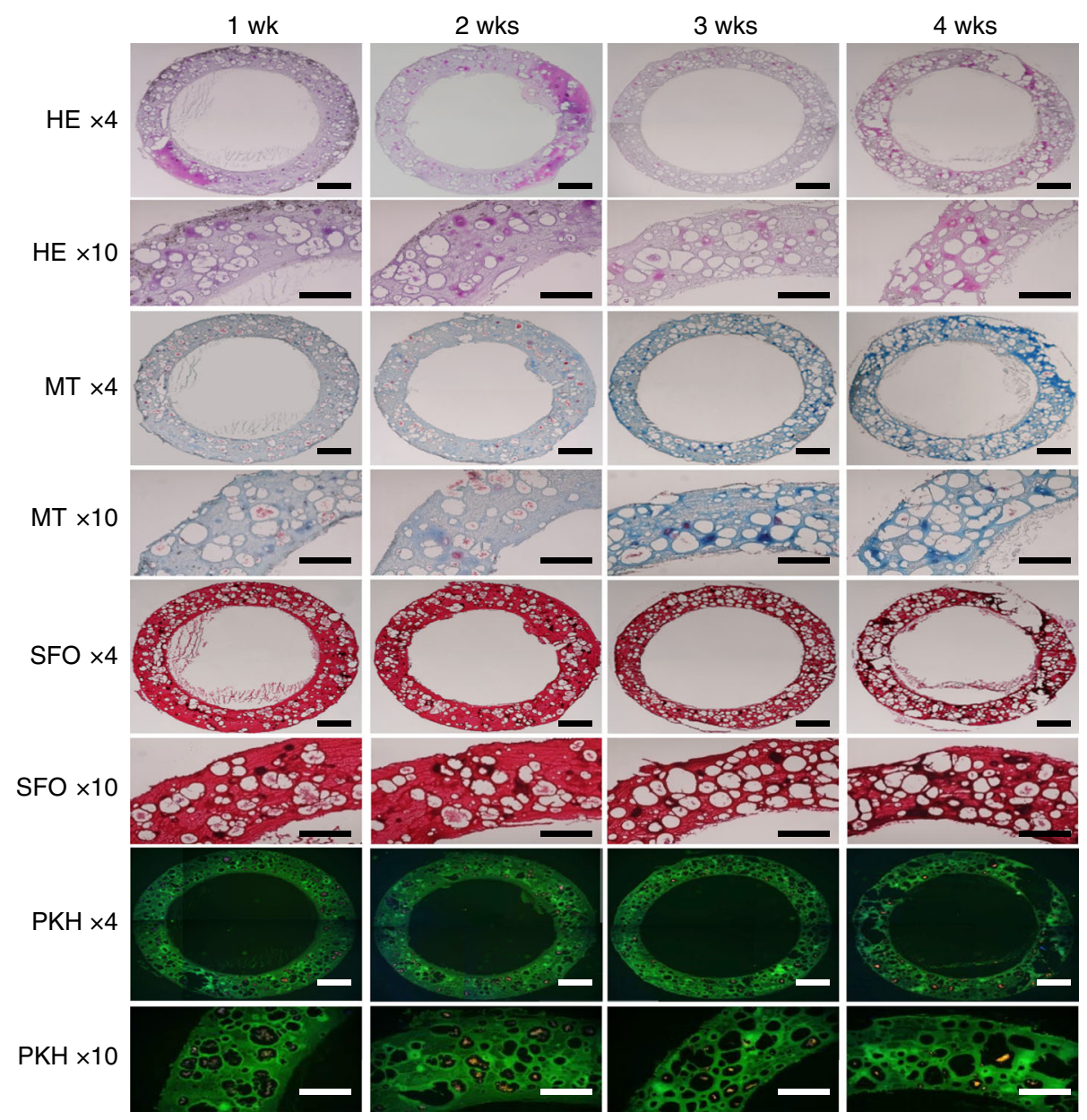

Fig. 7 In vitro histological evaluation of human chondrocytes loaded Sil-MA hydrogel for cartilage tissue engineering. Scale bars represent $1 \mathrm{~mm}(\times 4)$ and $500 \mu \mathrm{m}(\times 10)$. Sil-MA hydrogel showed superior histological characteristics (cell organization and extracellular matrix distribution, including proteoglycan and collagen) of cartilage-like tissue with time in vitro. HE H\&E, MT Masson's trichrome, SFO Safranin-O, PKH PKH26 (red: chondrocytes, green Sil-MA hydrogel)

It is worthy of note that Sil-MA bioink uses only naturally occurring polymers, rather than most of the alternatives, which are a combination of a natural polymer with a synthetic material $^{57,58}$, and yet the Sil-MA hydrogel produces equivalent strength and stability. This study suggests that photopolymerizable Sil-MA would be a superb bioink for DLP printing for biological and clinical applications.

\section{Methods}

Preparation of methacrylated SF powder and hydrogel prepolymer. B. mori cocoons were obtained from the Rural Development Administration (Jeonju, Korea). Each silkworm cocoon was sliced into four pieces. A unit of $40 \mathrm{~g}$ of sliced cocoons were boiled in $1 \mathrm{~L}$ of $0.05 \mathrm{M} \mathrm{Na}_{2} \mathrm{CO}_{3}$ solution for $30 \mathrm{~min}$ at $100{ }^{\circ} \mathrm{C}$ to remove the sericins, and then washed with distilled water several times. Figure $1 \mathrm{~b}$ is a schematic diagram illustrating the preparation and photopolymerization of the Sil-MA used for the DLP printer. Subsequently, degummed silk was dried at room temperature and $20 \mathrm{~g}$ of it was dissolved in $100 \mathrm{~mL}$ of $9.3 \mathrm{M}$ lithium bromide ( $\mathrm{LiBr}$ ) solution at $60^{\circ} \mathrm{C}$ for $1 \mathrm{~h}$. Right after SF was solved by $\mathrm{LiBr}, 2,4,6$, and $10 \mathrm{~mL}(141$, 282,424 , and $705 \mathrm{mM}$ ) of GMA solution (Sigma-Aldrich, St. Louis, USA) were added to the mixture stirring with a speed of $300 \mathrm{rpm}$ for $3 \mathrm{~h}$ at $60^{\circ} \mathrm{C}$ to create a high yield reaction between GMA and SF. Then, the resulting solution was filtered through a miracloth (Calbiochem, San Diego, USA) and dialyzed against distilled water using $12-14 \mathrm{kDa}$ cutoff dialysis tubes for 4 days. Finally, methacrylated SF solutions were frozen in $-80^{\circ} \mathrm{C}$ for $12 \mathrm{~h}$ and freeze-dried for $48 \mathrm{~h}$. Lyophilized methacrylated SF (Sil-MA) powder was stored $-80^{\circ} \mathrm{C}$ for further use.

Characterization (NMR/FT-IR/SEM). To determine the degree of methacrylation, the Sil-MA and varying amounts of GMA were examined through ${ }^{1} \mathrm{H}-\mathrm{NMR}$ at a frequency of $400 \mathrm{MHz}$ using a Bruker DPX FT-NMR Spectrometer $(9.4 \mathrm{~T})$ of
Bruker Analytik GmbH company (Karlsruhe, Germany) and $700 \mu \mathrm{L}$ deuterium oxide $\left(\mathrm{D}_{2} \mathrm{O}\right.$, Sigma-Aldrich) as the solvent per $5 \mathrm{mg}$ sample ${ }^{59}$. Sil-MA solution was filtered using a $0.45 \mu \mathrm{m}$ filter before analysis. ${ }^{1} \mathrm{H}$-NMR spectra were recorded for unsubstituted SF (RSF) and GMA with 1:0.7, 1:1.4, 1:1, and 1:3.4 mol unsubstituted amines on silk:mol GMA. Baseline correction was applied before measuring the integrals of the peaks of interest with ACD/NMR Processor Academic Edition. The degree of methacrylation was defined according to the percentage of $\varepsilon$-amino groups of lysine in SF that are modified in Sil-MA. For this, the signal from the protons produced by the aromatic amino acids in Sil-MA at 6.9-7.5 ppm was used to normalize each spectrum. Then, the lysine methylene signals $(2.8-2.95 \mathrm{ppm})$ of samples were integrated to obtain the areas. The degree of methacrylate substitution was determined by the formula: 1 - (lysine integration signal of Sil-MA/lysine integration signal of unsubstituted SF) $\times 100^{29}$. FT-IR spectra can be a valuable tool in studying graft copolymerization reactions. The structure of Sil-MA was confirmed by FT-IR spectra (Frontier, PerkinElmer, Rodgau, UK). Samples were prepared by grinding the samples with potassium bromide $(\mathrm{KBr})$. The microstructures of the Sil-MA hydrogels were observed under a field-emission SEM (VPFE-SEM; EVO ${ }^{\oplus S 10}$, Carl Zeiss, Germany) at the Korean Basic Science Institute (KBSI, Chuncheon, Korea). Printed Sil-MA hydrogel by 3D DLP printer was freeze-dried. The samples were coated with a thin $10 \mathrm{~nm}$ layer of gold/palladium for $30 \mathrm{~s}$ at $15 \mathrm{~mA}$ discharge current (Ion Sputter 1010, Hitachi, Japan). The micrographs were obtained at an accelerating voltage of $1.2-1.3 \mathrm{kV}$.

DLP 3D printing. Our photocurable hydrogel formulation was used in a DLP projector. Freeze-dried Sil-MA (prepared by $424 \mathrm{mM}$ of GMA) was dissolved in water at various concentrations of $10-30 \% \mathrm{w} / \mathrm{v}$ and the photoinitiator was added and mixed until fully dissolved. In these experiments, the photoinitiator, LAP (Tokyo chemical industry, Tokyo, Japan) was used at a concentration of $0.2 \% \mathrm{w} / \mathrm{v}$ in hydrogel prepolymer (Fig. 1b).

Over-curing of layers beyond the focal plane should be prevented to produce the best-quality $3 \mathrm{D}$ structures. To minimize this problem, a high-quality DLP 
printer of laboratory scale was designed and fabricated (Fig. 3a). Our DLP printing system consists of three major components: UV Digital Micro-mirror Device ${ }^{\mathrm{rt}}$ with a resolution of $30 \mu \mathrm{m}(1920 \times 1068 \times 1080$ pixels; Texas Instruments, Dallas, USA), $365 \mathrm{~nm}$ UV-LED (LG Innotec, Seoul Korea) with average $30 \mathrm{~mW} \mathrm{~cm}^{-2}$ intensity, and a lens module (focal length $60 \mathrm{~mm}$, aperture $\mathrm{f} / 2.8$, distortion $<80 \%$, offset $0 \%$, working distance $130 \mathrm{~mm}$, and field of view $28.8 \times 16.5 \mathrm{~mm}$ ) with two UV-grade biconvex lenses ( $24 \mathrm{~mm}$ diameter). The build area is $35(L) \times 20(W) \times 120(H) \mathrm{mm}$ with a layer thickness adjustable from 5 to $200 \mu \mathrm{m}$. The system was customized by professional manufacturers (NBRTech. Ltd, Chuncheon, Korea and Illuminaid. Ltd, , Seongnam, Korea).

Specimens were designed by CADian3D (IntelliKorea, Seoul, Korea) and were saved as a STereoLithography (STL) file. Then, STL files were sliced in the $Z$ direction. Finally, slices were projected by the projector for every layer to create the designed 3D morphology. Prints were carried out by repeating the process of projecting an image into the hydrogel followed by raising the $\mathrm{Z}$-stage. Printing parameters were used as follows: printing thickness, $50 \mu \mathrm{m}$; number of base layer, 3; curing time of base layer, $4 \mathrm{~s}$; number of buffer layer, 1; and curing time of buffer layer, $3 \mathrm{~s}$. Totally, we printed $40-200$ layers depending on test type. After printing, the printed object was briefly rinsed with deionized water to remove unreacted solution.

Physical properties. Specimens with $1 \times 1 \times 0.2-\mathrm{mm}^{3}$ rectangular shape were printed for water uptake ability measurement ${ }^{60}$. Briefly, samples were hydrated for $0.3,0.5,1,2,3,4$, and $5 \mathrm{~h}$ in $1 \times \mathrm{PBS}(\mathrm{pH} 7.4)$ at $37^{\circ} \mathrm{C}$. PBS was completely removed at each sampling time and the gels were weighed to obtain $W_{\text {swollen. }}$. Then the gels were lyophilized and measured the dried weight $\left(W_{\mathrm{dry}}\right)$. The ratio of water uptake from dried Sil-MA $(Q)$ can be calculated as following equation

$$
Q=\left(W_{\text {swollen }}-W_{\text {dry }}\right) / W_{\text {dry }} \times 100(\%)
$$

Expansion rate from DLP product by water, PBS, and medium was estimated by measuring the length of $X$ - and $Y$-axis based on initial status right after printing. Disc-shaped specimens with $2.5 \mathrm{~cm}$ in diameter and $2 \mathrm{~mm}$ thickness were printed for rheological analysis. The rheological properties of Sil-MA were measured at $25^{\circ}$ $\mathrm{C}$ using an Anton Paar MCR 302 (Anton Paar, Zofingen, Switzerland) rheometer. Peltier element and a thermostatic hood for temperature control were equipped in the rheometer and a UV source (SP-LED-1, $365 \mathrm{~nm}$, USHIO, Cypress, CA) was added for photocrosslinking to optimally prepare the hydrogel prepolymer. The measurements were performed utilizing a sandblasted-type geometry (PP25 s${ }^{-1}$, $25 \mathrm{~mm}$ in diameter) with a humidified atmosphere in the measuring chamber. The edge of the specimen and surfaces were covered with oil to avoid sample drying. Minimum torque oscillation was $0.5 \mathrm{nN} \mathrm{m}$ and maximum torque was $200 \mathrm{mN} \mathrm{m}$.

The dynamic storage $\left(G^{\prime}\right)$ and loss modulus $\left(G^{\prime \prime}\right)$ of materials were measured in oscillatory mode at $25^{\circ} \mathrm{C}$. In the amplitude sweep measurement, various amounts of Sil-MA with LAP $(0.2 \% \mathrm{w} / \mathrm{v})$ were tested for strain value of 0.01 to $1 \%$ by keeping the frequency constant at $10 \mathrm{rad} \mathrm{s}^{-1}$. In case of the frequency sweep measurement, the hydrogel materials were tested from 0.158 to $25.1 \mathrm{rad} \mathrm{s}^{-1}$ frequency range by keeping the shear strain constant at $1 \%$. For the UV-curing test depending on concentration of Sil-MA and LAP, oscillatory measurements were performed at a frequency of $1 \mathrm{rad} \mathrm{s}^{-1}$ and the target strain value of $0.1 \%$, which was found to be in the linear viscoelastic region for both the liquid and the gelled state of Sil-MA. The crossover points of $G^{\prime}$ and $G^{\prime \prime}$ were defined as the gelation points. UV light shined on the specimen on the glass plate (CTD600/UV) for $300 \mathrm{~s}$.

The compressive stress-strain curve of hydrogel was obtained by applying uniaxial compression force with a universal testing machine (QM100S, QMESYS, Gunpo, Korea) that was equipped with a $10 \mathrm{kgf}$ load cell in an unconfined environment (Supplementary Fig. 2a). Hydrogel disks with dimensions of $11.2 \mathrm{~mm}$ (diameter) and $10 \mathrm{~mm}$ (height) were studied in the compressive tests. A compression force was loaded at a displacement rate of $5 \mathrm{~mm} \mathrm{~min}^{-1}$ until the specimen broke to calculate the stress at failure and strain at failure. The tensile test was carried out on QMESYS with tensile jigs at a stretch velocity or $5 \mathrm{~mm} \mathrm{~min}^{-1}$ at room temperature in air, using a hydrogel specimen cut into the dumbbell shape of a concave column with measurements the size of length $16 \mathrm{~mm}(L)$ and width 7 $\mathrm{mm}(W)$ (Supplementary Fig. 2b). The sample thickness was $2 \mathrm{~mm}$. All specimens for mechanical testing were prepared via DLP printing. From the stress-strain curve, the secant modulus at 1,25 , and $50 \%$ strain for compressive test and at $50 \%$ for tensile test was calculated. All tests of physical properties were performed at strictly controlled condition room $\left(80 \%\right.$ humidification, $\left.27^{\circ} \mathrm{C}\right)$ to prevent drying out.

Hydrogel suturing and ex vivo study. A hydrogel membrane of $2.5 \mathrm{~cm} \times 2.5$ $\mathrm{cm} \times 0.15 \mathrm{~cm}(W \times L \times H)$ was printed out through DLP printer. This membrane was rolled out and sutured end to end. For ex vivo study, larynx and trachea from male beagle $\operatorname{dog}(15 \mathrm{~kg})$ was fixed with $4 \%$ formalin, which was donated from the animal center of Kangwon University (Korea). Trachea end to end anastomosis of larynx and trachea was carried out using DLP-printed Sil-MA trachea $(2.5 \mathrm{~cm} \times 2$ $\mathrm{cm} \times 1 \mathrm{~cm}: W \times L \times H)$. To suture hydrogel itself and between trachea and hydrogel, simple interrupted suture was applied using 3-0 coated VICRYL ${ }^{\circledast}$ (Ethicon, Johnson \& Johnson, USA).
Printability. Porous scaffolds and Eiffel Tower models with complex detail were printed by using $30 \%$ Sil-MA ( $424 \mathrm{mM} \mathrm{GMA}$ ) solution consisted with LAP $(0.2 \%$ w/v) through DLP printer. In addition, various organ shapes such as ear, brain, trachea, heart, lung, and blood vessel network were printed by using the same $30 \%$ Sil-MA bioink. After printing, the newly created ear and brain were pressed by an adult male's fingers ( 30 years old). To visualize the mechanical distortion of the $30 \%$ Sil-MA hydrogel-printed organs, compressive test was performed using a $7 \mathrm{~kg}$ kettle bell for $3 \mathrm{~min}$.

The actual resolution and accuracy of 3D DLP printing using Sil-MA. In order to check the actual resolution and similarity to the CAD files of the developed system, 30\%Sil-MA was 3D-printed into a block with gradually degrading circle or square patterns ranging from 900 to $70 \mu \mathrm{m}$ in the planar axis and from 900 to 200 $\mu \mathrm{m}$ in the vertical axis. The actual figure size was measured and the deviation was compared to the nominal sizes in both the horizontal and vertical planes.

Cell viability and proliferation. NIH/3T3 mouse fibroblast cells were purchased from Koram Biotech (ATCC distributor, Korea). Chondrocytes were isolated from human septal cartilage obtained from patients who underwent septoplasty, with informed consent. The procedures described in this study were approved by the Institutional Review Board of Chuncheon Sacred Heart Hospital of Hallym University (IRB No. 2017-87). The cartilage was washed several times with sterile PBS and chopped as finely as possible using a scalpel blade under aseptic conditions. Then the minced samples digested with $0.6 \%$ collagenase A (Roche Applied Science, Germany) at $37^{\circ} \mathrm{C}$ for $6 \mathrm{~h}$. Isolated chondrocytes were cultured into $10 \mathrm{~cm}$ culture dishes until confluent and sub-cultured up to two passages. The cell medium consists of Dulbecco's modified Eagle medium with $10 \% \mathrm{v} / \mathrm{v}$ fetal bovine serum and $1 \% \mathrm{v} / \mathrm{v}$ penicillin-streptomycin. All cultures were maintained in $5 \% \mathrm{CO}_{2}$ incubator at $37^{\circ} \mathrm{C}$ with the medium changed every 3 days. Cells are detached using trypsin-EDTA $0.25 \%$, counted, and prepared for the DLP printing. In all, $1 \times 10^{6}$ cells were mixed with Sil-MA solution containing LAP $(0.2 \% \mathrm{w} / \mathrm{v})$. Afterwards, this cell-loaded Sil-MA solution was used as bioink for DLP printing. The printed architectures were cultured for 14 days in the above culture medium. Cytotoxicity was evaluated using a Live/dead assay kit (Life Technologies, USA) according to the manufacturer's instruction. Samples were visualized and imaged using a fluorescent microscope (Eclipse 80i, Nikon, Tokyo, Japan) after $24 \mathrm{~h}$ incubation at $37^{\circ} \mathrm{C}$ and $5 \% \mathrm{CO}_{2}$. Cell proliferation in the cell-laden hydrogel was examined by CCK-8 assay (Dojindo Molecular Technology, Rockville, USA) according to the manufacturer's protocol.

Cell distribution in Sil-MA hydrogel. For live-cell imaging, NIH/3T3 cell line was stained with PKH26 and PKH67 (red and green fluorescence, Sigma-Aldrich) as the manufacturer's instruction. Cells were mixed with Sil-MA solution at a density of $2 \times 10^{7}$ cells per $\mathrm{mL}$ for red labeling and $1 \times 10^{7}$ cells per $\mathrm{mL}$ for green labeling. Samples $\left(4 \times 4 \times 4 \mathrm{~mm}^{3}\right)$, including a logo (Hallym University), a trachea, and brain were printed by DLP printer. Light sheet microscopy referred to as single plane illumination microscopy (SPIM) allowed to be obtained multidimensional imaging to be performed from several microns to several millimeters, minimizing photobleaching and phototoxic effect and enhancing image contrast and axial resolution The cell distribution in the 30\%Sil-MA hydrogel was detected using Lightsheet Z.1 microscope (Carl Zeiss Microscopy GmbH, Jena, Germany) with Lightsheet Z.1 detection optics $\times 5 / 0.16$ and two-sided Illumination Optics Lightsheet Z.1 $\times 5 / 0.1$ equipped with two detection module PCO at KBSI (Chuncheon, Korea). Edge cameras (sCMOS sensor, pixel size of $6.5 \mu \mathrm{m}, 1920 \times 1920$ pixel resolution, 15-bit depth, $30 \mathrm{fps}$ at $1000 \times 1000$ pixel). These samples were glued on the sample chamber in front of the detection lens. It was possible to rotate the sample and collect 3D stacks from multiple angles. One of the printed logos was observed by confocal microscope (Nanoscope, Korea).

Cartilage tissue engineering. 30\%Sil-MA solution containing $1 \times 10^{7}$ cells per mL of human chondrocytes was printed out to a trachea-shaped ring-like cartilage ( 7 $\mathrm{mm}$ of external diameter, $5 \mathrm{~mm}$ of internal diameter, and $6 \mathrm{~mm}$ of height) by DLP printer for cartilage tissue engineering. For in vitro test, the cell-loaded Sil-MA hydrogels were kept in a standard $\mathrm{CO}_{2}$ incubator for 4 weeks. All constructs were evaluated at each time point of $1,2,3$, and 4 weeks.

Histological evaluation. Samples from in vitro tests were fixed with $10 \%$ formaline for $24 \mathrm{~h}$, immersed in $30 \%$ sucrose for 1 day and molded with Tissue-Teck Optimum Cutting Temperature compound (Tissue Tek, Elkhart, IN, USA), and frozen in liquid nitrogen. Frozen samples were cut to a $10 \mu \mathrm{m}$ thickness, and encapsulated chondrocytes, general structure of tissue, and chondrogenesis were examined. To see the PKH26-labeled and encapsulated cells in hydrogel, sections were stained with Dapi (Vector Lab., Burlingame, USA) and observed under a fluorescence microscope (Eclipse 80i, Nikon Co., Japan). H\&E (HE) to assess tissue morphology, Safranin-O staining to identify the presence of proteoglycan-rich matrix, Masson's Trichrome (MT) staining to detect to collagen production were applied. Stained sections were analyzed under a microscope (Eclipse 80i, Nikon Co., Japan). 
Degradation study. From above samples, $10 \mu \mathrm{m}$-thickness sections were prepared without staining. The autofluorescence from SF was taken by a confocal imaging plate reader, InCell ${ }^{\circ}$ analyzer 2200 (GE Healthcare, Piscataway, USA) with a 475 $\mathrm{nm}$ excitation line and $525 \mathrm{~nm}$ band pass filter. The signal strength from the SilMA autofluorescence was measured by InCell ${ }^{\circ}$ developer toolbox after stitching pictures using InCell ${ }^{\oplus}$ analyzer workstation. The remaining Sil-MA was assessed as a percentage of the Sil-MA area at each time point versus the initial area of the SilMA hydrogel ${ }^{61}$.

Statistical analysis. Student unpaired (two-sample) $t$-test was used for statistical analysis. Data were presented as the mean value \pm standard deviation.

Data availability. The authors declare that all data supporting the findings of this study are available within the article and its Supplementary Information files or from the corresponding author on reasonable request.

Received: 4 July 2017 Accepted: 8 March 2018

Published online: 24 April 2018

\section{References}

1. Derby, B. Printing and prototyping of tissues and scaffolds. Science 338, 921-926 (2012).

2. Guillotin, B. \& Guillemot, F. Cell patterning technologies for organotypic tissue fabrication. Trends Biotechnol. 29, 183-190 (2011).

3. Murphy, S. V. \& Atala, A. 3D bioprinting of tissues and organs. Nat. Biotechnol. 32, 773-785 (2014).

4. Ozbolat, I. T. \& Yu, Y. Bioprinting toward organ fabrication: challenges and future trends. IEEE Trans. Biomed. Eng. 60, 691-699 (2013).

5. Zhu, W. et al. 3D printing of functional biomaterials for tissue engineering. Curr. Opin. Biotechnol. 40, 103-112 (2016).

6. Ma, X. et al. Deterministically patterned biomimetic human iPSC-derived hepatic model via rapid 3D bioprinting. Proc. Natl Acad. Sci. USA 113, 2206-2211 (2016).

7. Wang, Z. et al. A simple and high-resolution stereolithography-based 3D bioprinting system using visible light crosslinkable bioinks. Biofabrication 7 , 045009 (2015)

8. Keriquel, V. et al. In situ printing of mesenchymal stromal cells, by laserassisted bioprinting, for in vivo bone regeneration applications. Sci. Rep. 7, 1778 (2017).

9. Ouyang, L., Highley, C. B., Rodell, C. B., Sun, W. \& Burdick, J. A. 3D printing of shear-thinning hyaluronic acid hydrogels with secondary cross-linking. ACS Biomater. Sci. Eng. 2, 1743-1751 (2016).

10. Elomaa, L. et al. Three-dimensional fabrication of cell-laden biodegradable poly (ethylene glycol-co-depsipeptide) hydrogels by visible light stereolithography. J. Mater. Chem. B 3, 8348-8358 (2015).

11. Loebel, C., Rodell, C. B., Chen, M. H. \& Burdick, J. A. Shear-thinning and selfhealing hydrogels as injectable therapeutics and for 3D-printing. Nat. Protoc. 12, 1521 (2017).

12. Singh, M., Haverinen, H. M., Dhagat, P. \& Jabbour, G. E. Inkjet printingprocess and its applications. Adv. Mater. 22, 673-685 (2010).

13. Mandrycky, C., Wang, Z., Kim, K. \& Kim, D.-H. 3D bioprinting for engineering complex tissues. Biotechnol. Adv. 34, 422-434 (2016).

14. Grogan, S. P. et al. Digital micromirror device projection printing system for meniscus tissue engineering. Acta Biomater. 9, 7218-7226 (2013).

15. Lin, H. et al. Application of visible light-based projection stereolithography for live cell-scaffold fabrication with designed architecture. Biomaterials 34, 331-339 (2013).

16. Castro, N. J., O’Brien, J. \& Zhang, L. G. Integrating biologically inspired nanomaterials and table-top stereolithography for 3D printed biomimetic osteochondral scaffolds. Nanoscale 7, 14010-14022 (2015).

17. Axpe, E. \& Oyen, M. L. Applications of alginate-based bioinks in 3D bioprinting. Int. J. Mol. Sci. 17, 1976 (2016).

18. Müller, M., Becher, J., Schnabelrauch, M. \& Zenobi-Wong, M. Nanostructured Pluronic hydrogels as bioinks for 3D bioprinting. Biofabrication 7, 035006 (2015).

19. Shao, Z. \& Vollrath, F. Materials: surprising strength of silkworm silk. Nature 418, 741-741 (2002)

20. Nuttelman, C. R. et al. Macromolecular monomers for the synthesis of hydrogel niches and their application in cell encapsulation and tissue engineering. Progress Polym. Sci. 33, 167-179 (2008).

21. Lee, J. M. et al. Three dimensional poly ( $\varepsilon$-caprolactone) and silk fibroin nanocomposite fibrous matrix for artificial dermis. Mater. Sci. Eng. C 68 , 758-767 (2016).

22. Lee, O. J. et al. Fabrication and characterization of hydrocolloid dressing with silk fibroin nanoparticles for wound healing. Tissue Eng. Regen. Med. 13, 218-226 (2016).
23. Moon, B. M. et al. Novel fabrication method of the peritoneal dialysis filter using silk fibroin with urease fixation system. J. Biomed. Mater. Res. B Appl. Biomater. 105, 2136-2144 (2017).

24. Dal Pra, I., Freddi, G., Minic, J., Chiarini, A. \& Armato, U. De novo engineering of reticular connective tissue in vivo by silk fibroin nonwoven materials. Biomaterials 26, 1987-1999 (2005).

25. Park, H. J. et al. Fabrication of 3D porous silk scaffolds by particulate (salt/ sucrose) leaching for bone tissue reconstruction. Int. J. Biol. Macromol. 78, 215-223 (2015).

26. Park, Y. R. et al. Three-dimensional electrospun silk-fibroin nanofiber for skin tissue engineering. Int. J. Biol. Macromol. 93, 1567-1574 (2016).

27. Sheikh, F. A. et al. 3D electrospun silk fibroin nanofibers for fabrication of artificial skin. Nanomed. Nanotechnol. Biol. Med. 11, 681-691 (2015).

28. Murphy, A. R. \& Kaplan, D. L. Biomedical applications of chemicallymodified silk fibroin. J. Mater. Chem. 19, 6443-6450 (2009).

29. Nichol, J. W. et al. Cell-laden microengineered gelatin methacrylate hydrogels. Biomaterials 31, 5536-5544 (2010).

30. Yue, K. et al. Synthesis, properties, and biomedical applications of gelatin methacryloyl (GelMA) hydrogels. Biomaterials 73, 254-271 (2015).

31. Chen, Y. C. et al. Functional human vascular network generated in photocrosslinkable gelatin methacrylate hydrogels. Adv. Funct. Mater. 22, 2027-2039 (2012)

32. Xiao, W. et al. Synthesis and characterization of photocrosslinkable gelatin and silk fibroin interpenetrating polymer network hydrogels. Acta Biomater. 7, 2384-2393 (2011).

33. Kundu, J., Poole-Warren, L. A., Martens, P. \& Kundu, S. C. Silk fibroin/poly (vinyl alcohol) photocrosslinked hydrogels for delivery of macromolecular drugs. Acta Biomater. 8, 1720-1729 (2012).

34. Applegate, M. B. et al. Photocrosslinking of silk fibroin using riboflavin for ocular prostheses. Adv. Mater. 28, 2417-2420 (2016).

35. Lee, B. H., Shirahama, H., Cho, N.-J. \& Tan, L. P. Efficient and controllable synthesis of highly substituted gelatin methacrylamide for mechanically stiff hydrogels. RSC Adv. 5, 106094-106097 (2015).

36. Matsumoto, A. et al. Mechanisms of silk fibroin sol-gel transitions. J. Phys. Chem. B 110, 21630-21638 (2006).

37. Reis, A.V. et al. Reaction of glycidyl methacrylate at the hydroxyl and carboxylic groups of poly(vinyl alcohol) and poly(acrylic acid): is this reaction mechanism still unclear? J. Org. Chem. 74, 3750-3757 (2009).

38. Teramoto, N. et al. Preparation and mechanical properties of photocrosslinked fish gelatin/imogolite nanofiber composite hydrogel. Materials 5, 2573-2585 (2012).

39. Mondal, M. The silk proteins, sericin and fibroin in silkworm, Bombyx mori Linn.,-a review. Casp. J. Environ. Sci. 5, 63-76 (2007).

40. Li, X., Zhang, J., Kawazoe, N. \& Chen, G. Fabrication of highly crosslinked gelatin hydrogel and its influence on chondrocyte proliferation and phenotype. Polymers 9, 309 (2017).

41. Burtovyy, O. et al. Hydrophobic modification of polymer surfaces via "grafting to" approach. J. Macromol. Sci. B Phys. 46, 137-154 (2007).

42. Eckert, A. W., Gröbe, D. \& Rothe, U. Surface-modification of polystyrenemicrotitre plates via grafting of glycidylmethacrylate and coating of polyglycidylmethacrylate. Biomaterials 21, 441-447 (2000).

43. Fairbanks, B. D., Schwartz, M. P., Bowman, C. N. \& Anseth, K. S. Photoinitiated polymerization of PEG-diacrylate with lithium phenyl-2, 4, 6trimethylbenzoylphosphinate: polymerization rate and cytocompatibility. Biomaterials 30, 6702-6707 (2009).

44. Odian, G. In Principles of Polymerization 4th Edn (ed. Odian, G.) 198-349 (John Wiley \& Sons, Hoboken, 2004).

45. Suo, H., Xu, K. \& Zheng, X. Using glucosamine to improve the properties of photocrosslinked gelatin scaffolds. J. Biomater. Appl. 29, 977-987 (2015).

46. Engler, A. J., Sen, S., Sweeney, H. L. \& Discher, D. E. Matrix elasticity directs stem cell lineage specification. Cell 126, 677-689 (2006).

47. Kai, D. et al. Mechanical properties and in vitro behavior of nanofiber-hydrogel composites for tissue engineering applications. Nanotechnology 23, 095705 (2012).

48. Hasan, A. et al. Biomechanical properties of native and tissue engineered heart valve constructs. J. Biomech. 47, 1949-1963 (2014).

49. Floren, M., Migliaresi, C. \& Motta, A. Processing techniques and applications of silk hydrogels in bioengineering. J. Funct. Biomater. 7, 26 (2016).

50. Nagarkar, S., Nicolai, T., Chassenieux, C. \& Lele, A. Structure and gelation mechanism of silk hydrogels. Phys. Chem. Chem. Phys. 12, 3834-3844 (2010).

51. Möller, L. et al. Preparation and evaluation of hydrogel-composites from methacrylated hyaluronic acid, alginate, and gelatin for tissue engineering. Int. J. Artif. Organs 34, 93-102 (2011).

52. Miller, J. S. et al. Rapid casting of patterned vascular networks for perfusable engineered three-dimensional tissues. Nat. Mater. 11, 768-774 (2012).

53. Zhang, Y., Yu, Y. \& Ozbolat, I. T. Direct bioprinting of vessel-like tubular microfluidic channels. J. Nanotechnol. Eng. Med. 4, 020902 (2013).

54. $\mathrm{Wu}, \mathrm{Z}$. et al. Bioprinting three-dimensional cell-laden tissue constructs with controllable degradation. Sci. Rep. 6, 24474 (2016). 
55. Park, J. et al. Cell-laden 3D bioprinting hydrogel matrix depending on different compositions for soft tissue engineering: Characterization and evaluation. Mater. Sci. Eng. C 71, 678-684 (2017).

56. Peterson, G. I. et al. Production of materials with spatially-controlled crosslink density via vat photopolymerization. ACS Appl. Mater. Interfaces 8 , 29037-29043 (2016).

57. Schuurman, W. et al. Bioprinting of hybrid tissue constructs with tailorable mechanical properties. Biofabrication 3, 021001 (2011).

58. $\mathrm{Xu}, \mathrm{T}$. et al. Hybrid printing of mechanically and biologically improved constructs for cartilage tissue engineering applications. Biofabrication 5, 015001 (2012).

59. Brinkman, W. T., Nagapudi, K., Thomas, B. S. \& Chaikof, E. L. Photo-crosslinking of type I collagen gels in the presence of smooth muscle cells: mechanical properties, cell viability, and function. Biomacromolecules 4 , 890-895 (2003).

60. Chan, V., Zorlutuna, P., Jeong, J. H., Kong, H. \& Bashir, R. Three-dimensional photopatterning of hydrogels using stereolithography for long-term cell encapsulation. Lab Chip 10, 2062-2070 (2010).

61. He, Y., Dong, Y., Cui, F., Chen, X. \& Lin, R. Ectopic osteogenesis and scaffold biodegradation of nano-hydroxyapatite-chitosan in a rat model. PLoS ONE 10, e0135366 (2015).

\section{Acknowledgements}

This work was supported by the National Research Foundation of Korea (NRF) grant funded by the Korea government (MSIP; grant No.: 2016R1E1A1A01942120), by the Korea Health Technology R\&D Project through the Korea Health Industry Development Institute (KHIDI), funded by the Ministry of Health \& Welfare, Republic of Korea (grant No.: HI17C1229), and Hallym University research fund. We thank Prof. Jae Hyeon Ko (Department of Physics, Hallym University) for many helpful discussion about hydrogel's modulus. We also thank Dr. Seokbeom Kim (Illuminaid, Korea) for interpreting resolution of DLP printer.

\section{Author contributions}

C.H.P. conceived and supervised the study. G.K., S.J.L., and J.J.Y. gave advices about 3D printer, materials, and study design. S.H.K. collected and analyzed the data and wrote the paper with contributions from co-authors. Y.K.Y. led all the experiments with contributions from S.H.K., J.M.L., Y.J.L., and Y.B.S.; samples were printed out by Y.K.Y. and Y.J.L.; J.M.L sutured hydrogels. J.R.C. assisted in writing the manuscript and determined clinical applications from the results. Y.B.S. and M.T.S. performed biocompatibility test with contributions from I.H.; M.T.S. assisted in editing the manuscript and searching for the references. O.J.L. and J.S.L. supported experimental set-up. S.H.K., J.M.L., and S.i.Y. drew 2D/3D schematic illustration. All authors discussed the results and commented on the paper.

\section{Additional information}

Supplementary Information accompanies this paper at https://doi.org/10.1038/s41467018-03759-y.

Competing interests: The authors declare no competing interests.

Reprints and permission information is available online at http://npg.nature.com/ reprintsandpermissions/

Publisher's note: Springer Nature remains neutral with regard to jurisdictional claims in published maps and institutional affiliations.
Open Access This article is licensed under a Creative Commons Attribution 4.0 International License, which permits use, sharing, adaptation, distribution and reproduction in any medium or format, as long as you give appropriate credit to the original author(s) and the source, provide a link to the Creative Commons license, and indicate if changes were made. The images or other third party material in this article are included in the article's Creative Commons license, unless indicated otherwise in a credit line to the material. If material is not included in the article's Creative Commons license and your intended use is not permitted by statutory regulation or exceeds the permitted use, you will need to obtain permission directly from the copyright holder. To view a copy of this license, visit http://creativecommons.org/ licenses/by/4.0/.

(C) The Author(s) 2018 